\title{
Mechanistic Approach of Goss Abnormal Grain Growth in Electrical Steel: Theory
}

\section{and Argument}

Soran Birosca ${ }^{1}$, Ali Nadoum ${ }^{1}$, Diween Hawezy ${ }^{1}$, Fiona Robinson ${ }^{2}$, Winfried Kockelmann ${ }^{3}$

${ }^{1}$ Materials Research Centre, College of Engineering, Swansea University, Bay Campus, Swansea, SA1 8EN, UK.

${ }^{2}$ Cogent Power, Orb Electrical Steels, Newport, South Wales, NP19 ORB, UK.

${ }^{3}$ Science and Technology Facilities Council, Rutherford Appleton Laboratory, ISIS Facility, Harwell, OX11 0QX, UK.

\begin{abstract}
The first Si-Fe electrical steel was produced in 1905, and the grain-oriented steel was discovered in 1930 after Goss demonstrated how optimal combinations of heat treatment and cold rolling could produce a texture giving Si-Fe strip good magnetic properties when magnetised along its rolling direction. This technology has reduced the power loss in transformers greatly and remains the basis of the manufacturing process today. Since then many postulations reported on the mechanism on abnormal grain growth (AGG) which is the key for Si-Fe superior magnetic properties, however, none have provided a concrete understanding of this phenomenon. Here, we established and demonstrated a new theory that underlines the fundamental mechanistic approach of abnormal grain growth in $3 \%$ Si-Fe steel. It is demonstrated, that the external heat flux direction applied during annealing and $\mathrm{Si}$ atom positions in the solid solution disordered $\alpha-\mathrm{Fe}$ cube unit cell that cause lattice distortions and BCC symmetry reduction are the most influential
\end{abstract}


factors in the early stage of Goss AGG than what was previously thought to be dislocation related stored energy, grain boundary characteristics and grain size/orientation advantages.

Keywords: Abnormal Grain Growth, Electrical Steel, EBSD, Heat Flow, Goss Texture, Geometrically Necessary Dislocation, Neutron Texture Analysis.

\section{Introduction:}

Silicon steel was originally designed to replace iron-wrought alloy in transformers and the first commercial strip was produced in 1939 [1], after the Goss patent [2]. Henceforth, grain oriented electrical steel (GOES) has been widely used for transformer's core due to its excellent magnetic properties. The magnetic performance of GOES is highly dependent on the Goss $\{110\}<001>$ oriented grain that can abnormally grow during steel processing $[3,4]$. Thus, understanding the abnormal grain growth (AGG) of Goss grains is vital in reducing the power losses and increasing permeability in GOESs, that have great impacts on global power consumption and $\mathrm{CO}_{2}$ reduction [3]. To achieve the optimum physical properties in GOES, meticulous thermomechanical processing and annealing must be implemented. Commercially, GOES production starts from conventional steelmaking, then continuous casting, followed by hot rolling, hot band annealing and cold rolling. The cold rolled sheet is then decarburised, i.e., primary annealing, at $850^{\circ} \mathrm{C}$ in a wet hydrogen atmosphere. Final annealing to grow Goss grains abnormally, which is falsely socalled "secondary recrystallisation" is carried out at $1100-1200^{\circ} \mathrm{C}$ for $\sim 10$ days [4-6]. We strongly believe "Secondary Recrystallisation" should be replaced with "Secondary Annealing" in literature as the recrystallisation stage is already completed (or partially) in the primary annealing process and the grain growth is the main phenomenon in the secondary annealing stage. The secondary annealing process is responsible for eliminating the AIN grain growth inhibitors, which originally acted to pin the grain boundaries during primary annealing [7-9]. It is also believed that in the 
absence of these grain growth inhibitors, only normal grain growth occurs [10, 11]. However, the mechanism of AGG phenomena in which a specific orientation grows abnormally during secondary annealing is still disputed in literature and is the subject of this investigation.

The AGG for Goss oriented grains attracted numerous investigations and different mechanisms of AGG are reported [3, 7-32]. Some suggested the initial Goss grain size and orientation advantages over other orientations for AGG occurrence $[10,15,24]$. Others proposed that the coincidence site lattice (CSL) boundary promotes the AGG phenomenon $[16,25,26]$. They assumed that $\Sigma 7$ and $\Sigma 9$ boundaries have the highest mobility/energy and support Goss AGG. However, some argued the significance of CSL role in AGG and suggested a bigger role of high angle grain boundary (HAGB) misorientation of $\left(20^{\circ}-45^{\circ}\right)$ in Goss grain's AGG $[13,14,17,30,31]$. However, criticism of this concept grew when it was experimentally demonstrated that only a few grains underwent AGG, although more the $50 \%$ of the grain boundaries had $20-45^{\circ}$ misorientation angles $[11,19]$. Other researchers applied the solid-state wetting theory for Goss AGG [20-23, 32]. They claimed that if one of the grain boundaries around Goss grains has higher energy than the sum of the other two grain boundaries at the triple junction, the Goss grain can grow by liquid phase wetting in the direction of the highest energy boundary. Others theorised the AGG of Goss grains to be correlated to the stored energy via dislocation accumulation during cold rolling [33-37]. It is hypothesised that the Goss grains have low Taylor Factor values, i.e., low stored energy, and consumes the surrounding grains with high Taylor Factor values [5]. However, both latter postulations are also contested as many orientations other than Goss were found to have low Taylor Factor and satisfied the solid-state wetting condition, but they did not grow abnormally. Here, we examined most of these theories and proposed a new mechanism for Goss oriented grain AGG during secondary annealing. 


\section{Experimental Procedures:}

2.1 Materials: Commercial 3.2\% Si-Fe grain-oriented silicon steel was supplied by Cogent Orb in Newport-UK. Specimens through each processing stages were provided, including; hot rolled, cold rolled, primary annealed and secondary annealed samples. The chemical composition of the final stage supplied GOES is show in the Table 1.

Table 1: Chemical composition (wt.\%) of the final GOES product used in this study with the remaining of Fe.

\begin{tabular}{|c|c|c|c|c|c|c|c|c|c|c|}
\hline Si & $\mathbf{C}$ & $\mathbf{N}$ & $\mathbf{M n}$ & $\mathbf{P}$ & $\mathbf{A l}$ & $\mathbf{C u}$ & $\mathbf{N i}$ & $\mathbf{C r}$ & $\mathbf{S n}$ & $\mathbf{P b}$ \\
\hline 3.2 & 0.0017 & 0.0011 & 0.089 & 0.025 & 0.005 & 0.006 & 0.005 & 0.12 & 0.052 & 0.002 \\
\hline
\end{tabular}

2.2 Processing: All steel sheets were processed following standard Cogent Orb grain oriented electrical steel (GOES) industrial processing routes. Initially, Cogent Orb received the GOES from the Direct Sheet Plant where thin slabs are cast and passed directly into a tunnel furnace where a low reheat temperature of $\sim 1130^{\circ} \mathrm{C}$ is applied. The received sheets were then hot rolled to $2.3 \mathrm{~mm}$ thickness. A hot band is then annealed at $1050^{\circ} \mathrm{C}$ for a very short period before cold rolling. The decarburisation process, i.e., primary annealing, is performed at $850^{\circ} \mathrm{C}$ in a wet hydrogen atmosphere. After decarburising the strip passes into the nitriding section where ammonia is dissociated into $\mathrm{H}$ and $\mathrm{N}$ with the $\mathrm{N}$ being injected into the surface of the strip increasing the concentration from $70 \mathrm{ppm}$ to $250 \mathrm{ppm}$. The commercial secondary recrystallisation annealing was conducted at $1200^{\circ} \mathrm{C}$ in a dry protective atmosphere. The results reported here were from the interrupted and continuous primary and secondary annealing conducted at Swansea University laboratories and the in-situ annealing at the ISIS neutron diffraction facility at Harwell, UK.

2.3 Characterisation tools: Various nano/microanalytical tools were used in this study including Scanning Electron Microscope (SEM), Electron Backscatter Diffraction (EBSD) and neutron 
diffraction. For the microstructure investigation and characterisation, a JEOL 7800F Field Emission Gun Scanning Electron Microscopy (FEG SEM) equipped with an HKL EBSD and Oxford Energy Dispersive Spectroscopy (EDS) systems. The samples were prepared using 800, 1200 and 2000 grit grinding papers, followed by 5 min polishing using 6 and $3 \mu \mathrm{m}$ water-based diamond suspension. Colloidal silica suspension $0.04 \mu \mathrm{m}$ final polish was carried out for $15 \mathrm{~min}$. During EBSD data acquisition, the samples were tilted to $70^{\circ}$ and an acceleration voltage of $20 \mathrm{kV}$ was applied. The working distance was varied between 13 and $17 \mathrm{~mm}$. Furthermore, the Oxford Aztec software was used for EBSD and EDS mappings using a variant step size depending on the grain size and investigation purposes. The EBSD row data were then analysed using ATEX [41], HKL Channel 5 software and MATLAB-MTEX (MTEX is a free Matlab toolbox for analysing and modelling crystallographic textures by means of EBSD data) $[42,43]$. In-situ annealing was conducted using neutron diffraction facility at GEM (general materials diffractometer) beamline which is suitable for structure and texture analysis. The obtained data at different annealing temperatures were then analysed to generate Orientations Distribution Functions (ODF)s and calculate the precipitates volume fractions, lattice expansion and $\Delta d$-spacing variations from the peak shifting measurements. MATLAB-MTEX and MAUD software packages were used for peak fitting, peak position determination and lattice/d-spacing expansion calculations. MAUD (Material Analysis Using Diffraction) based on the Rietveld RITA/RISTA method, developed by L. Lutterotti, H.-R. Wenk, S. Matthies and others [44].

2.4 Annealing Trials: Various annealing trials were conducted for different investigation purposes including interrupted annealing and in-situ annealing. CARBOLITE furnace was used to study Goss abnormal grain growth behaviour at different annealing temperatures and time at Swansea University laboratories. Sample characterisation was carried out after cold rolling, hot rolling, commercial decarburisation annealing, laboratory annealing at various temperatures and times 
using different heating rates. In-situ annealing was conducted at GEM beamline (experiment

numbers: 1910029 and 1820052). The in-situ annealing was conducted using different heating rates while the neutron diffraction data were collected from $\mathrm{RT}$ up to $1100^{\circ} \mathrm{C}$. From the obtained diffraction peaks, the shift in diffraction peaks for lattice expansion calculations was measured and ODF plots throughout the annealing was recorded. The data were then used to determine the precipitates volume fractions during in-situ annealing of $3.2 \%$ Si steel.

\section{Results and Discussion:}

\subsection{Argument 1: Goss Grain Size and Orientation Advantages during AGG}

The first set of experiments was designed to validate the size and orientation advantages of Goss grains during AGG. Following the complete microstructure characterisations of a primary annealed specimen, the sample was then annealed interruptedly at $1030^{\circ} \mathrm{C} / 10 \mathrm{~s}$ and $1070^{\circ} \mathrm{C} / 8 \mathrm{~min}$. These selected temperatures were below the secondary annealing temperature in order to capture the early stage of grain growth. Figure 1a-c show the overall volume fraction (V.F \%) of each texture components. It should be noted that the data were obtained from a very large EBSD scan area of $7 \times 7 \mathrm{~mm}$, counting over 50,000 grains, see an example in Figure $1 \mathrm{~d}$. It is evident the $\gamma$-fibre V.F was the highest during all three annealing conditions. However, the Goss grain V.F was rather low even when is compared to Cube grains. Moreover, the Goss V.F unexpectedly decreased with increasing annealing temperature as illustrated in Figure 1a. In order to separate the early stage of normal grain growth with AGG, the V.F data were then sub-sectioned to grain sizes smaller and larger than $40 \mu \mathrm{m}$, in Figures $1 \mathrm{~b}$ and 1c, respectively. According to [19] the average grain size in a primary annealed sample is $\sim 20 \mu \mathrm{m}$ and they used a threshold of $40 \mu \mathrm{m}$ for large grains and initiation of AGG. This definition was also true is our case, see Figure $1 \mathrm{e}$, and $99 \%$ and $94 \%$ of the grains in the primary and $1070^{\circ} \mathrm{C}$ annealed samples, respectively, were $<40 \mu \mathrm{m}$. We, therefore, 
used the same threshold for AGG early stages in our calculations. A similar V.F progression trend

with annealing temperature was found for Goss and other grains $<40 \mu \mathrm{m}$ to that in (a). Nonetheless, this trend is changed by increasing V.F after $1030^{\circ} \mathrm{C}$ and reduced after $1070^{\circ} \mathrm{C}$ annealing for the Goss grains $>40 \mu \mathrm{m}$. This was a clear indication of the randomness of Goss oriented grains V.F evolution at the early stage of AGG. This random trend was conclusive for other orientations considering the overall and sub-sectioned data; thus, no preference was featured Goss grains at this stage of AGG.

This is further demonstrated via microstructure observation in Figure 1f. As shown in the figure, a selected area of a primary annealed sample was characterised by EBSD. The sample was then annealed at $1070^{\circ} \mathrm{C} / 8 \mathrm{~min}$, prepared metallographically again and EBSD scan is performed in the exact same area. The individual grain growth was observed using such a method and it appears there was no size preference of Goss grains as they have similar $20 \mu \mathrm{m}$ size in the primary annealed sample. It was also evident, that the Goss grains with a critical size, i.e., $>40 \mu \mathrm{m}$, in the primary annealed sample had completely disappeared and eliminated after the annealing at $1070^{\circ} \mathrm{C} / 8 \mathrm{~min}$, see the dashed blue and red areas in Figure 1f. Some Goss grains remained relatively constant in size, see Point A and some had grown dramatically from a non-Goss nucleus, see Point B. However, there is a possibility of Goss formation from a very fine Goss nucleus, but this cannot be verified due to the EBSD resolution limitation and 2D observation utilised here. Unexpectedly, the majority of the Goss grains that were larger than surrounding grains were either diminished or unchanged after annealing. Whereas, the majority of the increased grain size over $60 \mu \mathrm{m}$, in Figure 1e, originated from unpredicted areas where Goss was absent, see Figure 1f. We also examined the grain size distribution and it was clear from Figure 1e, no significant disparity in the grain size distribution was found between primary annealed and $1070^{\circ} \mathrm{C}$ samples. As expected, due to the grain growth, the grain size in the range of $10-20 \mu \mathrm{m}$ was reduced after 
the $1070^{\circ} \mathrm{C}$ annealing and an increase was observed for the grains $>20 \mu \mathrm{m}$. It is therefore concluded that the grain growth was not exclusive for Goss grains at the early stage of AGG as previously thought, as there was insignificant texture alteration after annealing. Moreover, the V.F and grain size for each texture components were randomly distributed following annealing trials.

\subsection{Argument 2: Grain Boundary (GB) and CSL effect on Goss Grains AGG}

In addition to the set of experiments discussed in Argument 1, two extra sets were conducted to examine GB and CSL boundary effects on AGG. For the sample annealed at 1030 and $1070^{\circ} \mathrm{C}$, the percentage of CSL boundaries did not change significantly as presented in Figure 2a. To validate this finding, the primary annealed sample was further annealed at 1000 and $1100^{\circ} \mathrm{C}$ for 20 min to observe the evolution at the later stage of AGG. The result was identical, see Figure $2 \mathrm{~b}$. This was a clear indication that CSL boundary evolution was rather random and no specific CSL increased remarkably. The small variations between different CSL percentages was rather dependant on the original microstructure and initial texture. The increased frequency of $\sum 9$ in Figure $2 b$ was due to its high amount in the original sample and its changes during annealing was negligible. The effect of grain boundary angle on grain growth was then investigated through characterising low $\left(<15^{\circ}\right)$ and high angle $\left(>15^{\circ}\right)$ grain boundaries, see Figure $2 \mathrm{c}$. It was clear that high angle grain boundaries (HAGB)s $>25^{\circ}$ in primary and secondary annealed samples at $1100^{\circ} \mathrm{C} / 20$ min were well fitted the Mackenzie random curve as indicated by the black lines over the bar charts. This grain boundary's random distribution was not changed during AGG even at $1100^{\circ} \mathrm{C}$.

Microstructurally, we identified all the Goss grains (blue) in the EBSD maps, see Figures $2 \mathrm{~d}_{1}$ and $2 \mathrm{e}_{1}$, in the primary annealed sample and plotted the GB's angles ranges values and CSL ( $\left.\sum 9-\sum 15\right)$ V.Fs (\%) surrounding each texture components, see Figures $2 \mathrm{~d}_{2}$ and $2 \mathrm{e}_{2}$. 
It is apparent from Figures $2 d_{1}$ and $2 e_{1}$, microstructurally, and $2 d_{2}$ and $2 e_{2}$, statistically, that Goss

grains show no distinct behaviour at this stage. The Goss grains, similar to other orientations were randomly surrounded by $<10^{\circ}, 10-20^{\circ}, 20-45^{\circ}$ and $45-60^{\circ}$ grain boundary misorientation angles. The $20-45^{\circ} \mathrm{GBs} \%$ V.F around Goss grains was even lower than that for Brass oriented grains in an EBSD scan area of over 10,000 grains; see Figure $2 \mathrm{~d}_{1-2}$. Clearly, this observation does not support the $20-45^{\circ} \mathrm{HAGB}$ effect on Goss grain AGG phenomenon as previously thought. A similar observation was found concerning CSL boundaries. As can be seen in Figure $2 \mathrm{e}_{1}$, microstructurally, and $2 \mathrm{e}_{2}$, statistically, the CSLs boundaries around Goss grains were rather random and in fact the $\sum 7$ and $\sum 9$ which thought to be responsible for Goss AGG, were lower than that for Cube, Copper and Rotated Cube oriented grains. Furthermore, the calculated energy differences between $\sum 5$, $\sum 7, \sum 9$, and $\sum 11$ in literature are very small and it is unreasonable to suggest that this insignificant energy dissimilarity is responsible for Goss growth in a range of a few millimetres or even centimetres in some cases. Finalising this argument, we can assertively state that grain boundary characteristics and CSL types around Goss grains have insignificant effect on Goss grain growth at the early stage of AGG.

\subsection{Argument 3: Dislocations, Stored Energy and Taylor Factor effects on Goss AGG}

A set of experiment was specifically designed to study the effect of deformation degree, dislocation density and stored energy on Goss AGG. A cold rolled 3.2\% Si steel (Figure 3a) was annealed interruptedly for 3, 4 and 5 minutes as presented in Figures $3 \mathrm{~b}-\mathrm{d}$. The calculated Geometrically Necessary Dislocation (GND) maps are shown beneath each IPF//RD maps and the average GND values for each texture component are presented in Figure 3f. The $\alpha$-fibre contains higher GND density than the $\gamma$-fibre in the cold rolled specimen, see Figure 3a $\mathrm{a}_{1} \mathrm{a}_{2}$. This has continued during subsequent annealing and the majority of GNDs were accumulated in the Rotated Cube texture component that is part of the $\alpha$-fibre, see Figure $3 b-d$ and $3 f$. It appears 
that the $\gamma$-fibre recovered (dislocation annihilation process) and recrystallised considerably faster than the $\alpha$-fibre during annealing. The GND maps/chart (in Figures 3a- $d$ and $f$ ) as well as the Tayler Factor (T.F) map/chart (Figure 3e and g), clearly show that GND and T.F values of different oriented grains are distributed and evolved rather randomly with annealing time. The Goss grains did not exhibit the lowest GND or T.F as previously believed in literature but were very similar to other orientations. The insignificant difference of Goss grains average GND and T.F values with other grains and its random changes during primary annealing cannot be responsible for AGG or early Goss growth.

To rationalise the assumption that Goss (low T.F and GND) surrounded by high GND and T.F grains is responsible of $A G G$, we plotted (Figure $3 e_{2}$ ) and calculated (Figure $3 h$ ) the Goss neighbouring grain volume fractions. It was evident that this assumption was not correct as reported in literature as the Goss grains were equally neighbouring high and low T.F grains irrespectively. Moreover, Goss grains seems to have larger numbers of Goss neighbours than Rotated Cube (high GND) or Rotated Goss, Cube and Copper (high T.F values). This set of experiments clearly showed that Goss's low Taylor Factor and GND can change during primary annealing and its differences with other orientations are insignificant for Goss AGG.

\subsection{Theory 1: Heat Directional Growth; Heat Flow Direction effects on Goss AGG}

Three different geometrical annealing configurations were designed to investigate the effect of heat flow and direction on Goss AGG: standard free flow heating (Figure 4a), direct exposure to heat flux on one side only (Figure $4 \mathrm{~b}$ ) and directional heating in a specific targeted angle (Figure 4c). From Figure 4a, it is clear that the AGG has occurred at both right (R) and left ( $L$ ) hand sides (HS) of the sample when exposed to heat flow on both sides. However, the AGG was not observed from the top and bottom sides of the samples as the furnace heating direction was horizontal 
along RHS and LHS directions, see the enlarged images in Figure 4a. A slight stronger AGG observed in the RHS, may be due to stronger external heat flux in this particular side and the furnace condition. However, for all the tested samples using this condition the AGG direction was observed to be parallel to heat flow direction. The $2^{\text {nd }}$ annealing condition was conducted by covering the RHS of the sample using a thermal insulator of Vermiculite treated silica cloth and Fiberfrax, see Figure $4 \mathrm{~b}$. It is clear from the figure and magnified images, that the AGG is only occurred in LHS of the sample and normal grain growth in the RHS. It appears that other orientations were grown on the edge of the sample's LHS, but only Goss grains finally won the competition and grew abnormally. The $3^{\text {rd }}$ condition was designed to monitor the AGG at specific angles of heating flow direction, an example is shown in Figure 4c. The AGG of Goss grain along (110) plane and the heating direction is evident when the sample was rotated by $45^{\circ}$ to control the Goss AGG direction. At the early stage of AGG, the Goss predomination among other orientations was visible in the magnified image in Figure 4c. Moreover, a few, but not all, Goss grains could grow abnormally along two (110) planes. This is well matched with FCC Silicon crystal growth direction along (110) plane and $(110)<100>$ orientation [38-40] and it appears to be also true for Fe BCC structure. The Goss AGG continues benefiting from grain size and orientation at this stage.

It is also established that the thermal conductivity increases with increasing grain size $[39,40]$ and this will further strengthen the continuation of Goss grain AGG. From the directional heating/AGG trials, we established that at the initial stage of AGG, Goss grains grew and annexed other oriented grains as the (110) planes expanded and grow dramatically when it was parallel to the heat flow direction depending on the heat flux amount. At the later stage of AGG, Goss was benefiting from its own orientation and size advantages as the thermal conductivity along (110) plane increased with increasing grain size. 
The temperature gradient across the overall sample is anticipated between the edge and the centre of the sample. However, considering the sample size and steel conductivity, the temperature gradient cannot play a significant part in AGG mechanism as the entire sample was heated well above AGG temperature. The faster growth rate of Goss grains at the edge of the sample compared to other oriented grains shown in Figures 4, demonstrate that temperature gradient within individual grains is more influential parameter for AGG to occur than overall sample temperature gradient. It appears that the temperature flux was the driving force for the Goss grain growth superiority to other oriented grains at the edge of the sample at the first nano second of the exposure, allowing Goss grains to advance early. Furthermore, the temperature as a single parameter was not the critical factor for Goss AGG, as only Goss grains grown abnormally, whilst all other orientations have grown normally which subjected to the same temperature at the edge of the sample. Moreover, only Goss grains grow abnormally when the heat flow was aligned with a particular $<110>$ as shown in Supplementary Figure S1. In S1, all 6 (110) slip trace was plotted for the maps shown in Figure 4. It is clear from the figure in S1 that the Goss grain growth direction was parallel to the heat flow direction and it is aligned perfectly with one or 2 of $\langle 110\rangle$ directions.

\subsection{Theory 2: Heating Rate and Precipitate Dissolution effect on Goss Grains AGG}

In-situ annealing using neutron diffraction was conducted to investigate the lattice expansion of $\mathrm{Fe}$ BCC (Figure 5a-b), and texture evolution (Figure 5c), and AIN precipitate V.F changes (Figure 5d), using different heating rates. The first set of neutron experiment was performed through heating the primary annealed GOES from RT up to $1070^{\circ} \mathrm{C}$ with a heating rate of $50^{\circ} \mathrm{C} / \mathrm{min}$. The diffraction data were obtained every 2 minutes. Figure 5a-b only show 4 sets of data, where the dramatic changes occurred during the in-situ test. The peak shift was calculated using original RT peak 
position as a reference point. The figure shows all Fe $d$-spacings have expanded gradually with

increasing temperature. However, the lattice expansion rate was reduced after $1020^{\circ} \mathrm{C}$. It is also evident from Figure $5 b$, that (110) $d$-spacing during BCC lattice expansion was the highest among all other planes, followed by (200). This (110) superior $d$-spacing expansion, has significantly maintained the growth of Goss grains along the heat flux direction during AGG as discussed in Theory 1 and will be demonstrated in Theory 3 (Section 3.6). Importantly, the high thermal conductivity in the (110) plane allowed the dissolution of AIN on Goss grain boundaries faster than in other orientations and promoted Goss AGG and success in the growth competition with other orientations. However, this type of thermal lattice expansion and grain growth need ultra clean materials with $0 \%$ precipitates as the precipitates can pin the grain boundary and restrict its movement. Thus, a second set of neutron experiments were conducted to measure AIN precipitates V.F \% and texture evolution using different heating rates. As can be seen in Figure 5c, the overall texture remained constant up to $1040^{\circ} \mathrm{C}$ using high $50^{\circ} \mathrm{C} / \mathrm{min}$ heating rate. At that point, the V.F of AIN precipitates was dropped due to sharp dissolution of AIN on the GBs, see Figure $5 \mathrm{~d}$. When the V.F of AIN reduced dramatically at $1070^{\circ} \mathrm{C}$ to 0.001 , a clear texture change was observed. The $\alpha^{*}$-fibre and $\gamma$-fibre (indicated on the ODFs) are completely disappeared at $1100^{\circ} \mathrm{C}$, where AGG of Goss grains is completed before final gradual elimination of AIN. It appears that using a fast heating rate, e.g., $50^{\circ} \mathrm{C} / \mathrm{min}$, dissolved the precipitates that pin the grain boundaries at high rate, and promoted AGG of Goss and other orientations, including Rotated Cube as shown in Figure 5c. This was further proved when we observed the texture changes during slower heating rate of $10^{\circ} \mathrm{C} / \mathrm{min}$ at $1030^{\circ} \mathrm{C}$ and complete disappearance on $\alpha^{*}$ fibre at $1040^{\circ} \mathrm{C}$ that lead to strong Goss texture generation at $1070^{\circ} \mathrm{C}$, see Figure $5 \mathrm{c}$. More gradual AIN elimination was achieved with a lower rate, up to $1040^{\circ} \mathrm{C}$ and a very sharp dissolution rate after $1040^{\circ} \mathrm{C}$; see Figure $5 \mathrm{~d}$, that facilitated a GB precipitates free for Goss AGG. It is therefore, 
recommended to perform secondary annealing at a low heating rate to produce stronger Goss texture.

It should be emphasized here that Figure $5 b$, shows the difference in $\Delta d$-spacing and not $d$-spacing where the lattice planes expansion was compared to a reference point (strain free at RT). It is well established that a uniform expansion of the cubic lattice leads to differential absolute expansions of the different $d$-spacings with (110) $d$-spacing increases more than the (002) d-spacing by a factor of 2.03/1.43. However, from $\Delta d$-spacing variation data in Figure $5 b$, it is clear that the materials transformed to a lower symmetry, as it is indicated by the peak shifting in Figure $5 \mathrm{a}$. To further clarify this further, $\Delta d / d$ versus temperature was plotted in Figure 6 , to check the validity of a higher-rate increase of $d(110)$ in absolute values. Figure 6a shows (110) expansion was similar to other planes up to $1070 \mathrm{C}$ where the Goss AGG is started. This is has led to strong Goss texture in the Si steel annealed using low heating rate, see Figure $5 c$. However, this is not the case, when the same material annealed at high heating rate, see Figure 6b. It is clear from the figure that (200) has the highest expansion rate at all temperatures, leading to strong Cube texture, see Figure $5 c$. This clearly indicates on the effect on particular lattice plane expansion ratio on texture development during AGG. As discussed previously this is dependent on alignment of specific plane direction along the heat flow to promote the expansion a particular lattice plane over others in the same crystal system. This is only valid if the BCC symmetry is reduced, which will be discussed in Section 3.6

\subsection{Theory 3: The effect of Si on $\alpha$-Fe lattice distortion}

In general, cubic crystalline materials including BCC and FCC structures, have equal thermal expansion coefficient in all three principle directions. It is also well established that thermal expansion and thermal conductivity are symmetric second rank tensor and not directionally 
dependant. Thus, here we assume that ideal Goss grain with prefect crystal structure, same as any

other oriented grains, is elastically expanding at high temperatures equally in all $\mathrm{X}, \mathrm{Y}$ and $\mathrm{Z}$ directions based on its BCC unit cell isotropic property. However, different lattice plane expansions within same unit cell as observed in Figure $5 b$ is crystallographic probability. It is believed that the BCC lattice is slightly distorted by the addition of Si atoms to $\alpha$-Fe unit cell in Fe3\%Si steel. The Si atoms that occupy substitutional sites in BCC unit cell, has $0.2 \mathrm{~nm}$ atom diameter, whereas $\alpha$-Fe has $0.248 \mathrm{~nm}$. This slightly smaller Si atoms make the Fe BCC lattice to be contracted according to $[45,46]$. The lattice parameter, $a_{\alpha}$, decreases with increasing Si content:

$\mathrm{a}_{\alpha}=0.2861-0.00015 \% \mathrm{Si}\left(\mathrm{a}_{\alpha}\right.$ in $\mathrm{nm}$ and $\mathrm{Si}<5$ wt.\%) [45]

According to the commonly accepted phase diagram of iron-silicon alloys, there are three basic phases $\left(\alpha, \alpha_{1}, \alpha_{2}\right)$. These phases are solid solution disordered (the case in this study with $3.2 \mathrm{wt} . \%$ Si) or with a short-range order $(\alpha)$, and structures with a long-range order of the DO3 type $\left(\alpha_{1}\right)$ and the B2 type $\left(\alpha_{2}\right)$ [47]. Although, the addition of Si in BCC iron was investigated by many researchers to improve the magnetic property of Si steel, however, the effect of Si atoms on lattice distortion is rarely studied. Instead most studies focused on nano structural materials or ordered structures in order to study the magnetic properties with very high Si content [48-50]. Nonetheless, they established the fact that with increasing Si contents, the lattice distortion increases. Moreover, F. Lin, et al. [51] claimed that with increasing temperature up to $500^{\circ} \mathrm{C}$, the (220) peak has shifted from $44.8^{\circ}$ at RT to $45.3^{\circ}$ at $500^{\circ} \mathrm{C}$. N. Overman. et al., [52], studied Fe-Si (38 wt.\%) physical property, and they demonstrated that with increasing Si content, the lattice distortion increased and caused peak shifting even without altering the $\alpha$-Fe BCC crystal structure. Similar finding was reported in 4.9 -5.8\% Si single crystal $\alpha$-Fe in [53]. 
It should be emphasized here that this different plane expansions reported here and in literature

is not necessarily to be observed as an overall bulk expansion in the three principle directions. The (110) lattice expansion superiority over the other lattice planes demonstrated in the current study is also observed in BCC beta titanium with addition of hydrogen as a solid solution of the BCC structure [54]. S. Shantilal [55] also used X-ray diffraction to measure thermal lattice expansion of Fe BCC at low temperatures. He claimed that the influence of impurities on Fe lattice parameter increases with increasing temperatures. S. Kim, et al [56] claimed that the thermal expansion coefficient of Fe BCC is very low, however, the volume variation may be considerable. Moreover, Z. Feng [57] applied Vegard's law [58] and concluded that the unit cell lattice parameter of an alloy, at a given temperature, changes linearly with the concentration of the substitutional elements. In general, depending on the substitutional element, the lattice expansion was observed in $\alpha$-Fe at various temperature ranges. Figure 7 show the lattice parameter increased with increasing temperature, which is in agreement with the previous literature. It is recognised that the elements in the periodic table to the left of Fe, expand $\alpha$-Fe lattice spacing due to their bigger sizes compared with Fe atoms. However, the elements in the periodic table to the right of $\mathrm{Fe}$, that are smaller than Fe atoms (the case of $\mathrm{Si}$ ), also displayed a lattice expansion due to the exchange repulsion between nearly filled $d$ shells $[57,59]$. It is postulated that with decreasing atoms distance, the electron clouds approach each other further, and their electronic charge distributions overlap. This leads to a reduction of electron density between the atoms due to the Pauli exclusion principle [59]. The atoms nuclei that are positively charged will not then be shielded completely from each other and create a repulsive force between them, causing lattices expansion [57-59]. This is also in agreement with Figure 8 where Si atoms is smaller in size compared with Fe atoms.

Rationally, we think there are two major evidences of the influence of Si atoms on Goss AGG: 
(i) Goss grains do not grow abnormally or preferentially in other alloys including $\mathrm{Ni}, \mathrm{Cu}, \mathrm{Al}$ alloys as well as BCC steels with no Si and Al contents (Chemical Composition and Lattice Structure Factors).

(ii) Not all Goss grains in the same deformation band in the cold rolled as well as in the primary annealed electrical steel sheets can grow abnormally (Selective Goss AGG).

This leads us to emphasise that the Si atom position in Fe BCC is a key in Goss AGG in Si Steel, see Figure 8. Thus, we further considered the effect of Si content on the peak shifting observed in the current study, Figure 5a. It was clear from Figure 7 that the lattice expansion, i.e., lattice parameters, increased with increasing temperature. It was also evident that the $d$-spacing of the individual crystallographic lattice planes were changed differently, see Figures $5 \mathrm{~b}$ and 6 . It should be emphasised here, this type of peak shifting along with peak broadening is also reported in other BCC structure during high temperature exposure $[60,61]$ and polycrystalline FCC [62]. It should be also remembered that (110) has a larger $d$-spacing (2.323 Angstrom) in an ideal $\alpha$-Fe BCC structure than that for (100) at RT [63] and it appears here that its $\Delta d$-spacing increased with increasing temperatures (especially above $1070^{\circ} \mathrm{C}$ ) at higher rate than other planes, see Figure $5 \mathrm{~b}$.

Further observation of Figure 5a revealed that peaks splitting, and broadening occurred after $1070^{\circ} \mathrm{C}$, where dramatic AGG starts. Some areas of the diffraction peak in Figure 5a and beyond $1070^{\circ} \mathrm{C}$ are enlarged for further clarifications. For instance, Figure 9, show peak boarding of (110) peak from initial FWHM of 0.002147 at RT to $0.018126 \mathrm{~A}$ at $1070^{\circ} \mathrm{C}$. The raw data with simple peak fitting is shown in the same figure. Figure 10 shows (110) peak partial splitting that occurred at $1070^{\circ} \mathrm{C}$ and further progressed at $1100^{\circ} \mathrm{C}$ and $1200^{\circ} \mathrm{C}$. The figure shows the critical breaking points on the (110) peak that are formed and increased with increasing temperature, indicating on further split of the (110) peak with increasing temperature. It should be emphasized here, only 
partial and not fully peak split observed in the current study. Furthermore, the peak splitting was

also apparent in (200) peak as shown in Figure 11. It is well established that peak broadening might be correlated to strain, dislocation density, grain size as well as symmetry reduction [64, 65]. However, it our case, the material was fully annealed, thus, most of the peak broadening may be related to grain size changes during grain growth as well as BCC symmetry reduction at high temperatures. It is also well established that, peak splitting is an indication of phase transformation to a lower symmetry. For example, from cubic to tetragonal, or from tetragonal to orthorhombic $[66,67]$. These observations indicate that at $1070^{\circ} \mathrm{C}$, where the dramatic AGG started, the Goss grain did not grow symmetrically, and it changed to much lower BCC symmetry when further annealed to $1100^{\circ} \mathrm{C}$ and $1200^{\circ} \mathrm{C}$. It should be noted that peak splitting can also occurs as a result of substructure and sub boundary formation as reported in [68]. However, the data obtained in the current study was above AGG condition for 3\% Si steel where the grains were fully recrystallised, and the Goss grain grown abnormally, thus no sub boundary could be created in such a condition.

From our basic Si atomic position calculations, we assume that in the disordered solid solution $\alpha$ Fe cubic structure if Si atoms occupy 2 or more of the 8 corners (e.g., $(1,1,1$ and $(0,0,0)$ cubic atomic positions), replacing Fe atoms substitutionally, see Figure $8 c$, this atomic configurations will make 2 out of the $6(110)$ plane $d$-spacings slightly even greater than that for other $4(110),(001)$ and other principle planes. However, if Si atom occupy only the centre position $(1 / 2,1 / 2,1 / 2)$ or 1 or 2 non-opposite corners of the $\alpha$-Fe cubic unit cell, the superiority of (110) $\Delta d$-spacing variation will not occur at the very high temperatures, see the atomic configurations in Figures $8 \mathrm{a}$ and $8 \mathrm{~b}$. Thus, it is reasonable to assume that not all the Goss grains in Fe-3\% Si steel can abnormally grow in the same sheet or the deformation band unless Si atoms occupy the right positions to unbale only 2 of the 6 (110) planes expansion possible. This clearly explains the Goss grain AGG in two of (110) 
planes in Figure 4c. In fact, we strongly believe that the lattice distortion occurrence due to Si

additions in a disordered $\alpha$-Fe unit cell, provides a great crystallographic advantage for Goss grain AGG. However, further study and appropriate atomic simulation are needed to identify the exact Si atoms position in $\alpha$-Fe unit cell that can satisfy the preferable lattice distortion and atomic configurations for Goss AGG.

\subsection{Closing Theory for AGG of Goss Orientation}

Figure 12 depicts the Goss AGG mechanism in GOES. Initially the addition of Si contents into $\alpha$-Fe unit cell can distort the ideal BCC lattice parameters in favour of 1 or 2 of the 6 (110) planes expansion and superior $\Delta d$-spacing during thermal exposure. Based on the Si atoms position and configuration in $\alpha$-Fe BCC unit cell, not all Goss grains can grow abnormally. Thus, only limited number of Goss grains will satisfy the (110) superior $\Delta d$-spacing condition and can grow abnormally, consuming other orientations including other Goss grains with unfavourable unit cell configurations as described in Section 3.6. In the early stage of AGG 2 or less of the 6 (110) planes have the highest expansion among other crystallographic planes which results in highest $\Delta d$ spacing. This assists greatly in accelerating the heat flux along (110) planes to reach the Goss GB first and dissolve AIN faster than in any other orientations. This clean, i.e., precipitate free, GB increases Goss GB mobility in the heat flow and growth directions to win the growth competition with other oriented grains. At a later stage of AGG, as the thermal conductivity increases with increasing grain size, the Goss grain grows dramatically along (110) planes where the heat flows rapidly in the Goss growth direction.

\section{Conclusions:}

We concluded that Goss oriented grains do not have size, orientation and grain boundary characteristic advantages during the early stage of AGG as they grew in a very similar manner to 
other orientations (Figures 1-3). However, at the later stage of AGG the large Goss grain increases

the thermal conductivity as they have proportional correlation (Figure 4). The dislocation accumulation, stored energy and T.F values are not correlated to Goss AGG (Figure 3) but rather to recrystallisation stage. It is demonstrated here, that the Goss AGG mechanism can be theorised via heat flow directional control of the grain growth (Figures 4 and 12) and lattice distortion via $\mathrm{Si}$ addition to $\alpha$-Fe BCC unit cell (Figures 5-8). From in-situ trials using neutron diffraction it was evident that the heating rate has a direct influence on the texture control through retarding and advancing the dissolution of precipitates that effect Goss AGG. For manufacturing and industrial benefits, it is recommended to use a low heating rate to obtain sharp Goss texture to reduce power loss in power transformation. Finally, the heat flow induced Goss AGG, will require a change in the current GOES manufacturing route if the concept is industrialised.

\section{Acknowledgements}

Special thank for AIM (Advanced Imaging of Materials) in Swansea University and Cogent Power for materials supply. Experiments at the ISIS Neutron and Muon Source were supported by a beam time allocation RB1910029 from the Science and Technology Facilities Council.

\section{References:}

[1] A.J. Moses, Electrical steels. Past, present and future developments, IEE Proc. A Phys. Sci. Meas. Instrumentation. Manag. Educ. Rev. 137 (1990) 233-245. doi:10.1049/ip-a-2.1990.0039. [2] N.P. Goss, Electrical sheet and method and apparatus for its manufacture and test, US Patent 1965559. (1934) 1-11.

[3] Y. Ushigami, M. Mizokami, M. Fujikura, T. Kubota, H. Fujii, K. Murakami, Recent development of low-loss grain-oriented silicon steel, J. Magn. Magn. Mater. 254 (2003) 307-314. 
[4] M. Yabumoto, S. Arai, R. Kawamata, M. Mizokami, T. Kubota, Recent development in grainoriented electrical steel with low magnetostriction, J. Mater. Eng. Perform. 6 (1997) 713-721.

[5] S.M. Shin, S. Birosca, S.K. Chang, B.C. De Cooman, Texture evolution in grain-oriented electrical steel during hot band annealing and cold rolling, J. Microsc. 230 (2008) 414-423.

[6] Z. Xia, Y. Kang, Q. Wang, Developments in the production of grain-oriented electrical steel, J. Magn. Magn. Mater. 320 (2008) 3229-3233. doi:10.1016/j.jmmm.2008.07.003.

[7] F. Fang, Y. Zhang, X. Lu, Y. Wang, G. Cao, G. Yuan, Y. Xu, G. Wang, R.D.K. Misra, Inhibitor induced secondary recrystallization in thin-gauge grain oriented silicon steel with high permeability, Mater. Des. 105 (2016) 398-403. doi:10.1016/j.matdes.2016.05.091.

[8] A. Morawiec, On abnormal growth of Goss grains in grain-oriented silicon steel, Scr. Mater. 64 (2011) 466-469. doi:10.1016/j.scriptamat.2010.11.013.

[9] X. Lu, F. Fang, Y.X. Zhang, Y. Wang, G. Yuan, R.D.K. Misra, W.N. Zhang, G.D. Wang, Secondary recrystallization behavior in strip-cast grain-oriented silicon steel processed by isothermal secondary annealing, Mater. Charact. 142 (2018) 68-76. doi:10.1016/j.matchar.2018.05.029.

[10] M. Hillert, On the theory of normal and abnormal grain growth, Acta Metall. 13 (1965) 227-238. doi:10.1016/0001-6160(65)90200-2.

[11] A. Morawiec, Grain misorientations in theories of abnormal grain growth in silicon steel, Scr. Mater. 43 (2000) 275-278. doi:10.1016/S1359-6462(00)00403-6.

[12] F. Citrawati, M.Z. Quadir, P.R. Munroe, Investigation on the Early Stages of Growth of Secondary Grains in a Grain Oriented Silicon Steel, Procedia Eng. 184 (2017) 750-755.

[13] C. Su, G. Zhao, H. Xiao, Y. Lan, F. Huang, Abnormal Grain Growth of Hi--B Steel in the Secondary Recrystallization, Metallogr. Microstruct. Anal. 7 (2018) 608-617. doi:10.1007/s13632018-0467-9. 
[14] Y. Hayakawa, J.A. Szpunar, A new model of Goss texture development during secondary

recrystallization of electrical steel, Acta Mater. 45 (1997) 4713-4720.

[15] J.E. Burke, D. Turnbull, Recrystallization and grain growth, Prog. Met. Phys. 3 (1952) 220292. doi:https://doi.org/10.1016/0502-8205(52)90009-9.

[16] R. Shimizu, J. Harase, Coincidence grain boundary and texture evolution in Fe-3\%Si, Acta Metall. 37 (1989) 1241-1249. doi:10.1016/0001-6160(89)90118-1.

[17] B. Ralph, K.B. Shim, Z. Huda, J. Furley, M.J. Edirisinghe, The effects of particles and solutes on grain boundary migration and grain growth, in: Mater. Sci. Forum, 1992: pp. 129-140.

[18] V. Randle, B. Ralph, Interactions of grain boundaries with coherent precipitates during grain growth, Acta Metall. 34 (1986) 891-898. doi:10.1016/0001-6160(86)90062-3.

[19] N. Chen, S. Zaefferer, L. Lahn, K. Günther, D. Raabe, Effects of topology on abnormal grain growth in silicon steel, Acta Mater. 51 (2003) 1755-1765. doi:10.1016/S1359-6454(02)00574-8.

[20] K.-J. Ko, P.-R. Cha, D. Srolovitz, N.-M. Hwang, Abnormal grain growth induced by subboundary-enhanced solid-state wetting: Analysis by phase-field model simulations, Acta Mater. 57 (2009) 838-845.

[21] K.-J. Ko, J.-T. Park, J.-K. Kim, N.-M. Hwang, Morphological evidence that Goss abnormally growing grains grow by triple junction wetting during secondary recrystallization of Fe- $3 \% \mathrm{Si}$ steel, Scr. Mater. 59 (2008) 764-767. doi:https://doi.org/10.1016/j.scriptamat.2008.06.021.

[22] N.M. Hwang, Simulation of the effect of anisotropic grain boundary mobility and energy on abnormal grain growth, J. Mater. Sci. 33 (1998) 5625-5629.

[23] H.-K. Park, S.-D. Kim, S.-C. Park, J.-T. Park, N.-M. Hwang, Sub-boundaries in abnormally growing Goss grains in Fe-3\% Si steel, Scr. Mater. 62 (2010) 376-378. doi:https://doi.org/10.1016/j.scriptamat.2009.11.025.

[24] C.G. Dunn, Secondary recrystallization textures and their origin in cold-rolled single crystals of silicon iron, Acta Metall. 1 (1953) 163-175. doi:10.1016/0001-6160(53)90055-8. 
[25] J. Harase, R. Shimizu, Coincidence grain boundary and (100)[011] secondary

recrystallization in Fe-3\% Si, Acta Metall. Mater. 40 (1992) 1101-1111. doi:10.1016/09567151(92)90088-V.

[26] J. Harase, R. Shimizu, D.J. Dingley, Texture evolution in the presence of precipitates in Fe3\% Si alloy, Acta Metall. Mater. 39 (1991) 763-770. doi:10.1016/0956-7151(91)90276-7.

[27] N. Rouag, G. Vigna, R. Penelle, Evolution of local texture and grain boundary characteristics during secondary recrystallisation of Fe-3\% Si sheets, Acta Metall. Mater. 38 (1990) 1101-1107. [28] J. Harase, R. Shimizu, J.-K. Kim, J. SooWoo, The role of high energy boundaries and coincidence boundaries in the secondary recrystallization of grain-oriented silicon steel, Met. Mater. 5 (1999) 429-435.

[29] N.C. Pease, D.W. Jones, M.H.L. Wise, W.B. Hutchinson, SEM study of origin of Goss texture in Fe-3.25 Si, Met. Sci. 15 (1981) 203-209.

[30] Y. Hayakawa, J.A. Szpunar, The role of grain boundary character distribution in secondary recrystallization of electrical steels, Acta Mater. 45 (1997) 1285-1295.

[31] A.L. Etter, T. Baudin, R. Penelle, Influence of the Goss grain environment during secondary recrystallisation of conventional grain oriented Fe-3\%Si steels, Scr. Mater. 47 (2002) 725-730. doi:https://doi.org/10.1016/S1359-6462(02)00189-6.

[32] K.J. Ko, P.R. Cha, J.T. Park, J.K. Kim, N.M. Hwang, Abnormal Grain Growth of Fe-3\%Si Steel Approached by Solid-State Wetting Mechanism, Adv. Mater. Res. 26-28 (2007) 65-68. doi:10.4028/www.scientific.net/AMR.26-28.65.

[33] L. Kestens, J.J. Jonas, Modeling texture change during the static recrystallization of interstitial free steels, Metall. Mater. Trans. A. 27 (1996) 155-164.

[34] J.K. Kim, J.S. Woo, S.K. Chang, Influence of annealing before cold rolling on the evolution of sharp Goss texture in Fe-3\%Si alloy, J. Magn. Magn. Mater. 215 (2000) 162-164. doi:10.1016/S0304-8853(00)00103-7. 
[35] S.H. Choi, Y.S. Jin, Evaluation of stored energy in cold-rolled steels from EBSD data, Mater.

Sci. Eng. A. 371 (2004) 149-159. doi:10.1016/j.msea.2003.11.034.

[36] S.F. Castro, J. Gallego, F.J.G. Landgraf, H.-J. Kestenbach, Orientation dependence of stored energy of cold work in semi-processed electrical steels after temper rolling, Mater. Sci. Eng. A. 427 (2006) 301-305. doi:https://doi.org/10.1016/j.msea.2006.04.092.

[37] S.K. Chang, Texture change from primary to secondary recrystallization by hot-band normalizing in grain-oriented silicon steels, Mater. Sci. Eng. A. 452-453 (2007) 93-98. doi:https://doi.org/10.1016/j.msea.2006.10.118.

[38] Y. Zhou, Y. Chen, M. Hu, Strong Surface Orientation Dependent Thermal Transport in Si Nanowires, Sci. Rep. 6 (2016) 24903. doi:10.1038/srep24903.

[39] Z. Aksamija, I. Knezevic, Anisotropy and boundary scattering in the lattice thermal conductivity of silicon nanomembranes, Phys. Rev. B - Condens. Matter Mater. Phys. 82 (2010) 45319. doi:10.1103/PhysRevB.82.045319.

[40] C.W. Nan, R. Birringer, Determining the Kapitza resistance and the thermal conductivity of polycrystals: A simple model, Phys. Rev. B - Condens. Matter Mater. Phys. 57 (1998) 8264-8268. doi:10.1103/PhysRevB.57.8264.

[41] B. Beausir, J.J. Fundenberger, Analysis tools for electron and X-ray diffraction, ATEXSoftware, Univ. Lorraine-Metz. (2017).

[42] F. Bachmann, R. Hielscher, P.E. Jupp, W. Pantleon, H. Schaeben, E. Wegert, Inferential statistics of electron backscatter diffraction data from within individual crystalline grains, J. Appl. Crystallogr. 43 (2010) 1338-1355. doi:10.1107/S002188981003027X.

[43] R. Hielscher, H. Schaeben, A novel pole figure inversion method: Specification of the MTEX algorithm, J. Appl. Crystallogr. 41 (2008) 1024-1037. doi:10.1107/\$0021889808030112. 
[44] L. Lutterotti, S. Matthies, H.R. Wenk, A.S. Schultz, J.W. Richardson, Combined texture and structure analysis of deformed limestone from time-of-flight neutron diffraction spectra, J. Appl. Phys. 81 (1997) 594-600. doi:10.1063/1.364220.

[45] B.C. De Cooman, J.G. Speer, I.Y. Pyshmintsev, N. Yoshinaga, Materials Design: The Key to Modern Steel Products, GRIPS media, 2007.

[46] N.I. Kulikov, D. Fristot, J. Hugel, A. V. Postnikov, Interrelation between structural ordering and magnetic properties in bcc Fe-Si alloys, Phys. Rev. B - Condens. Matter Mater. Phys. 66 (2002) 142061-142068. doi:10.1103/PhysRevB.66.014206.

[47] N. V Ershov, Y.P. Chernenkov, V.A. Lukshina, V.I. Fedorov, X-ray diffraction studies of specific features in the atomic structure of Fe-Si alloys in the alpha area of the phase diagram, Phys. Solid State. 51 (2009) 441-447.

[48] L. Zhiqiang, L. Helie, L. Wuyan, Z. Qingqi, The effect on Si on the local magnetic moment and hyperfine interaction of BCC-Fe, J. Phys. Condens. Matter. 3 (1991) 6649-6659. doi:10.1088/0953-8984/3/34/010.

[49] S.M. Dubiel, W. Zinn, Influence of Si on spin and charge density changes in bcc-iron, J. Magn. Magn. Mater. 28 (1982) 261-276. doi:10.1016/0304-8853(82)90058-0.

[50] M. Yousefi, S. Sharafi, The effect of simultaneous addition of Si and Co on microstructure and magnetic properties of nanostructured iron prepared by mechanical alloying, Mater. Des. 37 (2012) 325-333. doi:https://doi.org/10.1016/j.matdes.2012.01.011.

[51] F. Lin, D. Jiang, X. Ma, W. Shi, Structural order and magnetic properties of Fe3Si/Si(100) heterostructures grown by pulsed-laser deposition, Thin Solid Films. 515 (2007) 5353-5356. doi:10.1016/j.tsf.2007.01.024.

[52] N.R. Overman, X. Jiang, R.K. Kukkadapu, T. Clark, T.J. Roosendaal, G. Coffey, J.E. Shield, S.N. Mathaudhu, Physical and electrical properties of melt-spun Fe-Si (3-8 wt.\%) soft magnetic ribbons, Mater. Charact. 136 (2018) 212-220. doi:10.1016/j.matchar.2017.12.019. 
[53] Y.P. Chernenkov, V.I. Fedorov, V.A. Lukshina, B.K. Sokolov, N. V. Ershov, Short-range order in $\alpha$-Fe-Si single crystals, J. Magn. Magn. Mater. 254-255 (2003) 346-348. doi:10.1016/S03048853(02)00845-4.

[54] O.N. Senkov, B.C. Chakoumakos, J.J. Jonas, F.H. Froes, Effect of temperature and hydrogen concentration on the lattice parameter of beta titanium, Mater. Res. Bull. 36 (2001) 1431-1440. doi:10.1016/S0025-5408(01)00604-3.

[55] J.S. Shah, Thermal lattice expansion of various types of solids, Dr. Diss. 2312.Missouri Univ. Sci. Technol. (1971).

http://scholarsmine.mst.edu/doctoral_dissertations\%0Ahttp://scholarsmine.mst.edu/doctoral_dis sertations/2312.

[56] S.W. Kim, H. Tanigawa, T. Hirose, K. Shiba, A. Kohyama, Effects of surface morphology on fatigue behavior of reduced activation ferritic/martensitic steel, J. Nucl. Mater. 367-370 A (2007) 568-574. doi:10.1016/j.jnucmat.2007.03.238.

[57] Z. Feng, the Lattice Parameter of Gamma Iron and Iron-Chromium Alloys, Case Western Reserve University, 2015.

https://etd.ohiolink.edu/!etd.send_file?accession=case1429138602\&disposition=inline.

[58] A.R. Denton, N.W. Ashcroft, Vegard's law, Phys. Rev. A. 43 (1991) 3161.

[59] S. Brdarski, G. Karlström, Modeling of the exchange repulsion energy, J. Phys. Chem. A. 102 (1998) 8182-8192. doi:10.1021/jp9819893.

[60] H. Cao, F. Bai, J. Li, D.D. Viehland, T.A. Lograsso, P.M. Gehring, Structural studies of decomposition in Fe-x at.\%Ga alloys, J. Alloys Compd. 465 (2008) 244-249. doi:10.1016/j.jallcom.2007.10.080.

[61] A. Goldbach, L. Yuan, H. Xu, Impact of the $\mathrm{fcc} / \mathrm{bcc}$ phase transition on the homogeneity and behavior of PdCu membranes, Sep. Purif. Technol. 73 (2010) 65-70.

doi:10.1016/j.seppur.2010.01.007. 
[62] Y. Zhao, J. Zhang, Microstrain and grain-size analysis from diffraction peak width and

graphical derivation of high-pressure thermomechanics, J. Appl. Crystallogr. 41 (2008) 1095-1108. doi:10.1107/S0021889808031762.

[63] H.D. Omar, To Investigation the Structure and Morphology of Iron Metallic by Difference Techniques, J. Nano. Adv. Mat. 3 (2015) 57-61.

[64] E. Schafler, K. Simon, S. Bernstorff, P. Hanák, G. Tichy, T. Ungár, M. J. Zehetbauer, A secondorder phase-transformation of the dislocation structure during plastic deformation determined by in situ synchrotron X-ray diffraction, Acta Materialia

53, Issue 2 (2005) 315-322.

[65] I. S. Golovin, A. M. Balagurov, V. V. Palacheva, I. A. Bobrikov, V. B. Zlokazov, In situ neutron diffraction study of bulk phase transitions in Fe-27Ga alloys, Materials \& Design 98 (2016) 113-119.

[66] H.-R. Wenk, I. Huensche, L. Kestens, In-Situ Observation of Texture Changes during Phase Transformations in Ultra-Low-Carbon Steel, Metallurgical and Materials Transactions A 38 Issue 2 (2007) 261-267.

[67] Y. Tomota, P. G. Xu, T.Kamiyama, E. C. Oliver, In situ TOF neutron diffraction during phase transformation in an engineering steel, Nuclear Instruments and Methods in Physics Research Section A: Accelerators, Spectrometers, Detectors and Associated Equipment, Volume 600, Issue 1 (2009) 313-315.

[68] Tae-Young Kim, Hyung-Seok Shim, Seunggyu Kim, Sungki Choi, Tae-Wook Na, Dongil Kwon, Nong-Moon Hwang, Synchrotron X-ray microdiffraction analysis of abnormally growing grains induced by indentation in Fe-3\%Si steel, Materials Characertization, Volume 156 (2019) 109845. 


\section{Table Captions:}

Table 1: Chemical composition (wt.\%) of the final GOES product used in this study with the remaining of Fe.

\section{Figure Captions:}

Figure 1: The volume fraction (\%) of each texture components (Cube, Goss, Brass, Copper, $\alpha$-fibre, $\gamma$-fibre) in primary, $1030^{\circ} \mathrm{C} / 10$ s and $1070^{\circ} \mathrm{C} / 8 \mathrm{~min}$ annealed specimens in (a), for only the grain size $<40 \mu \mathrm{m}$ in (b) and for the grains $>40 \mu \mathrm{m}$ in (c). The graphs show that Goss grain (average as well as $40 \mu \mathrm{m}>$ Goss $>40 \mu \mathrm{m}$ sizes) were behaved similarly to other orientations during early stages of AGG and they do not have size or orientation advantages. (d) EBSD Invers Pole Figure (IPF) // Rolling Direction (RD) map of primary annealed sample showing an example of the large EBSD data set used for the statistical volume fraction calculations to plot the graphs in (a-c). (e) showing grain size distribution of both primary and $1070^{\circ} \mathrm{C} / 8 \mathrm{~min}$ annealed samples. (f) EBSD texture component maps for the same area that is marked in the primary and $1070^{\circ} \mathrm{C}$ annealed samples to observe the evolution of each oriented grains during early stage of $A G G$, the same colour specification for each texture components is used in the graph in (a-c) and (f) for comparison purposes.

Figure 2: Overall volume fraction (\%) of CSL boundaries for primary, $1030^{\circ} \mathrm{C} / 10 \mathrm{~s}$ and $1070^{\circ} \mathrm{C} / 8 \mathrm{~min}$ annealed specimens in (a) and for primary, $1000^{\circ} \mathrm{C} / 20 \mathrm{~min}$ and $1100^{\circ} \mathrm{C} / 20 \mathrm{~min}$ annealed specimens in (b). Grain boundary distribution of primary and $1100^{\circ} \mathrm{C} / 20 \mathrm{~min}$ annealed samples is shown in (c), the Mackenzie plot (thick black curve) is fitted the HAGBs indicating of their random distribution. The Goss oriented grains are highlighted in "Blue" within the EBSD grain boundary map in $\left(d_{1}\right)$ and CSL boundary Map in $\left(\mathrm{e}_{1}\right)$. The grain boundary misorientation degree and CSL type volume fraction 
(\%) surrounding each orientation are plotted in $\left(d_{2}\right.$ and $\left.e_{2}\right)$ showing their random distribution

around Goss and other oriented grains. Area 1 and Area 2 indicated in $\left(d_{1}\right.$ and $\left.e_{1}\right)$ are magnified to show examples of CSL and GBs types boundaries around Goss grains.

Figure 3: $\left(a_{1}, b_{1}, c_{1}, d_{1}\right)$ show EBSD Inverse Pole Figure IPF// Rolling direction (RD) for cold rolled, annealed for 3 minutes, 4 minutes and 5 minutes, respectively. $\left(a_{2}, b_{2}, c_{2}, d_{2}\right)$ show the calculated GND maps for the EBSD maps in $\left(a_{1}, b_{1}, c_{1}, d_{1}\right)$. The $\left(\mu \mathrm{m} / \mu \mathrm{m}^{3}\right)$ unit for GND density is used. The GND maps showed a speedy recovery and recrystallisation of $\gamma$-fibre compared to $\alpha$-fibre. $\left(e_{1}\right)$ shows EBSD calculated Taylor Factor map for $\left(b_{1}\right) .\left(e_{2}\right)$ shows the Tayler Factor maps for only the grains that neighbouring Goss grains. This map shows the Goss grains are surrounded randomly with high and low Taylor Factor grains. (f) shows the average GND dislocation density values for each texture components for the samples annealed at $850^{\circ} \mathrm{C}$ for 3 minutes, 4 minutes and 5 minutes. (g) shows the average Taylor Factor values for each texture components for the samples annealed at $850^{\circ} \mathrm{C}$ for 3 minutes, 4 minutes and 5 minutes. (h) shows statistical distribution of Goss neighbouring grain volume fractions (\%). The graph shows that the Goss grains have more Goss neighbours than Cube, Rotated Cube, Rotated Goss and Brass and Copper grains.

Figure 4: Three different geometrical annealing configurations: (a) standard free flow heating on both RHS and LHS of the sample, (b) direct exposure to heat flux only on one side of the sample, (c) directional heat targeted in a $45^{\circ}$ angle to the sample. In all cases the heat flow was parallel to Goss growth direction, i.e., (110) planes. Magnified images are shown for both RHS and LHS in (a) and (b), but only for LHS in (c) as the RHS in (c) was identical to the RHS in (b) where only normal grain growth occurred. 
Figure 5: The diffraction data obtained during in-situ annealing at ISIS neutron diffraction facility

from RT up to $1070^{\circ} \mathrm{C}$ using primary annealed GOES material is shown in (a). A magnified (110) peak is shown beside (a) to signify the lattice expansion ( $d$-spacing changes) at high temperatures. The change in lattice parameters, i.e., interplanar $d$-spacing in Angstrom, with temperature is shown in (b) for different lattice planes. (c) showing the texture evolution with temperature at different heating rates, the texture represented in $\varphi 2=45$ Orientation Distribution Function (ODF). (d) showing the evolution of AIN precipitates with temperature in a table, the dissolution rate vs. temperature is also plotted for different heating rates.

Figure 6: $\Delta d / d$ versus temperature graphs during in situ annealing using (a) low heating rate of $\left(10^{\circ} \mathrm{C} / \mathrm{min}\right)$ and $(\mathrm{b})$ high heating rate of $\left(50^{\circ} \mathrm{C} / \mathrm{min}\right)$.

Figure 7: The lattice parameter (a) in cubic BCC vs. temperature calculated using neutron diffraction data during in-situ thermal exposure trails. The figure shows the lattice parameters increased with increasing annealing temperatures.

Figure 8: $\left(a_{1}, b_{1}, c_{1}\right)$ and $\left(a_{2}, b_{2}, c_{2}\right)$ showing Fe (brown balls) and Si (grey balls) atomic configurations of disordered solid solution $\alpha$-Fe cubic unit cell in (100) and (110) plane views, respectively. ( $\left.a_{1}, a_{2}\right)$ showing small Si atom occupying the substitutional Fe atom in the centre position $(1 / 2,1 / 2,1 / 2) .\left(b_{1}, b_{2}\right)$ showing small Si atom occupying the substitutional Fe atom in one corner $(1,1,1) .\left(c_{1}, c_{2}\right)$ showing small Si atoms occupying the substitutional Fe atoms in $(1,1,1)$ and $(0,0,0)$ cubic atomic positions.

Figure 9: (110) peak broadening occurrence at high temperatures. The FWHM unit is in Angstrom. The broadening mostly correlated to the Goss grain growth and BCC symmetry reduction. 
Figure 10: (110) peak splitting at $1070^{\circ} \mathrm{C}$ and progressed at $1100^{\circ} \mathrm{C}$ and $1200^{\circ} \mathrm{C}$. The critical breaking points on the (110) peak are shown, indicating on partial split of the (110) peak with increasing temperature. Goss grain did not grow symmetrically, and it changed to lower BCC symmetry when further annealed to $1100^{\circ} \mathrm{C}$ and $1200^{\circ} \mathrm{C}$.

Figure 11: (200) peak splitting and broadening indicating on the reduction of BCC symmetry.

Figure 12: The mechanism of Goss AGG in GOES. The initiation of AGG is shown in GOES during secondary annealing at $1070^{\circ} \mathrm{C} / 8 \mathrm{~min}$.

\section{Supplementary Caption:}

S1: Slip Trace Maps $(a, b, c)$ for the EBSD maps shown in Figure 4 ( $b$ and $c$ ). The Goss abnormal grain growth was parallel to heat flow and one or $2<110>$ directions. 
(a) Volume fraction for each Texture Components

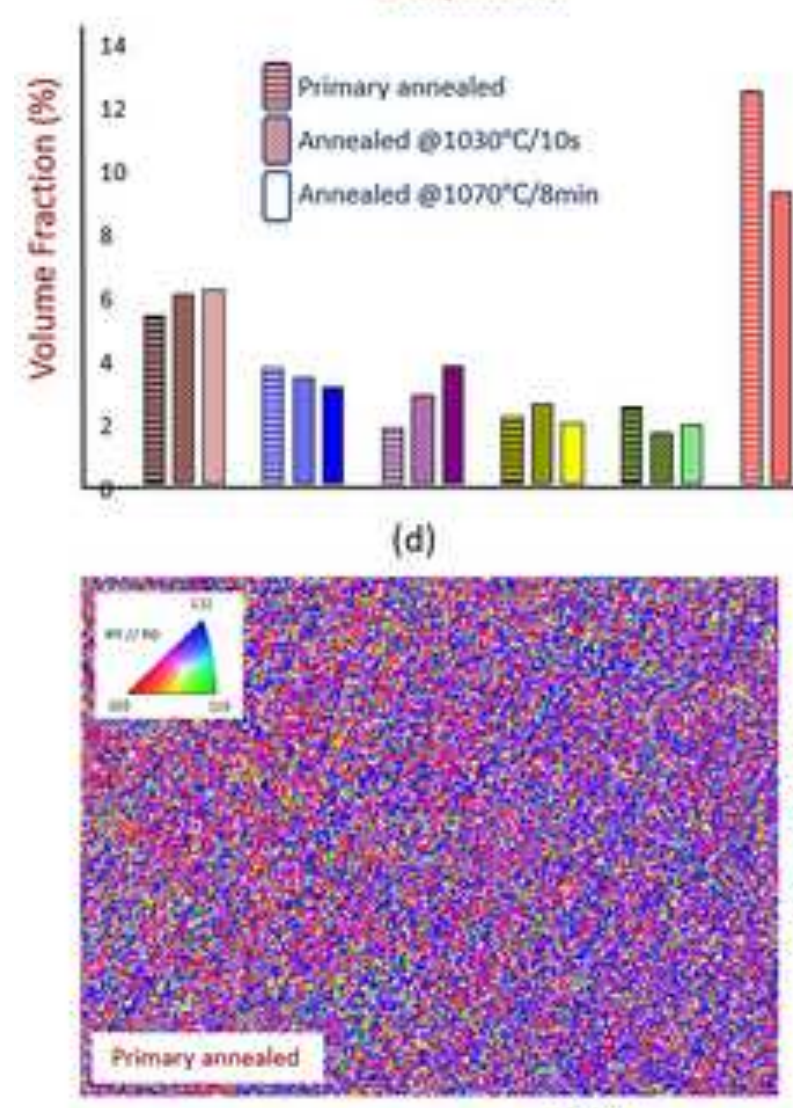

$2 \mathrm{~mm}$

Grain size $<40 \mu \mathrm{m}$ for each Texture

(c)
(b)

Components

Texture Components

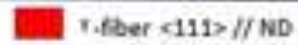

- fiber $\leqslant 1100 / / \mathrm{RD}$

$\square$ (a) (112)<111>

E. 1100$)<1123$

- $\cos (110), 0013$

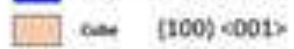

${ }_{4}^{6}$ 目

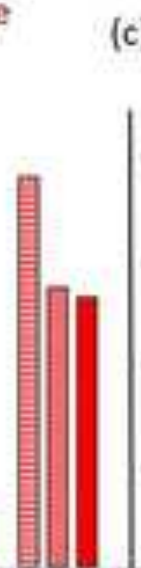

il:

\section{(e)}

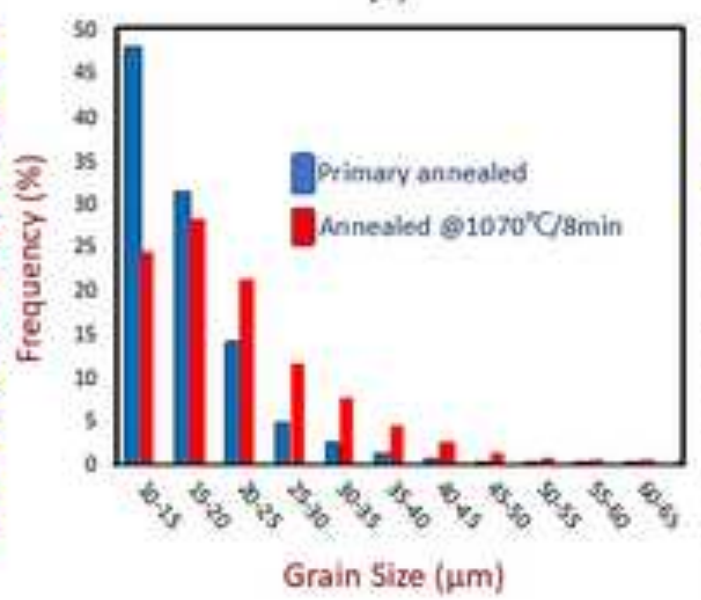

Primary annealed

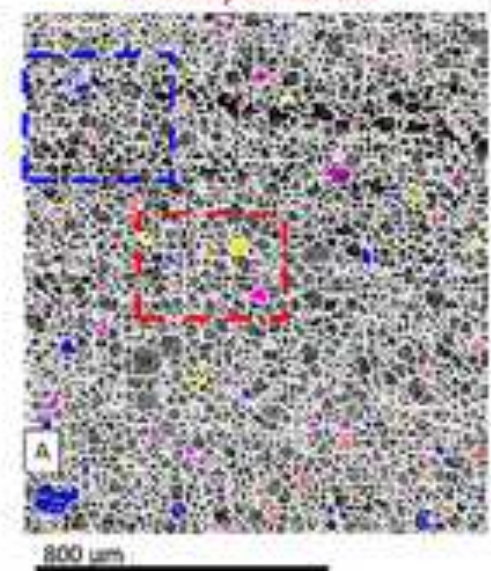

Grain size $>40 \mu \mathrm{m}$ for each Texture

Components

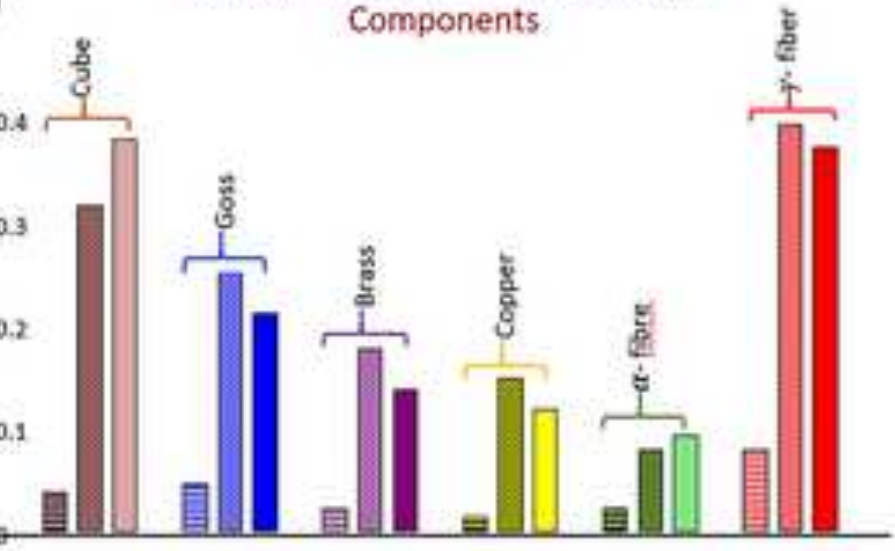

(f)

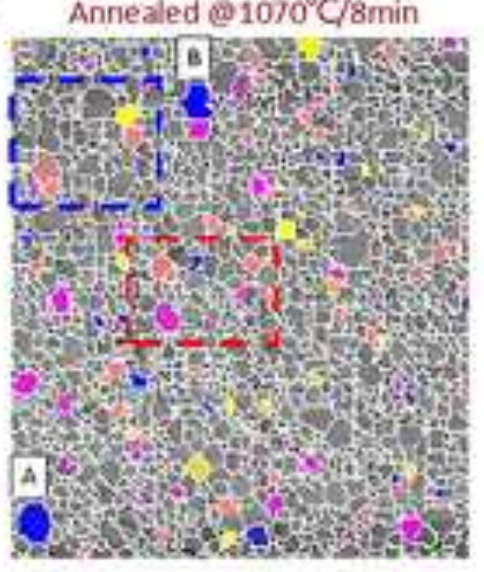




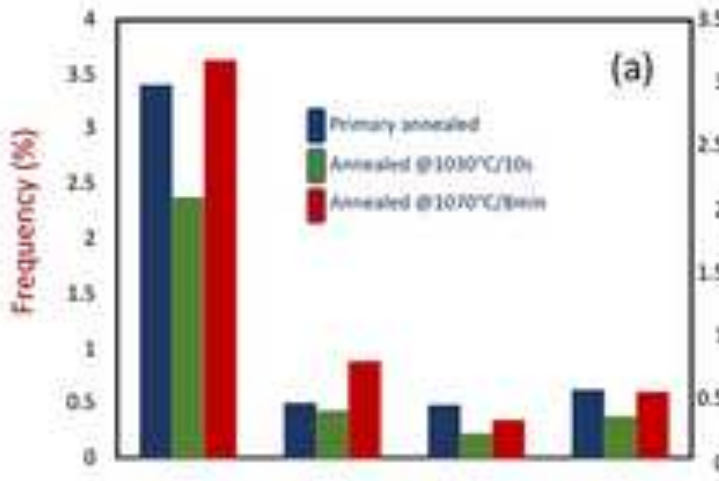

23

25

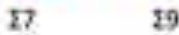

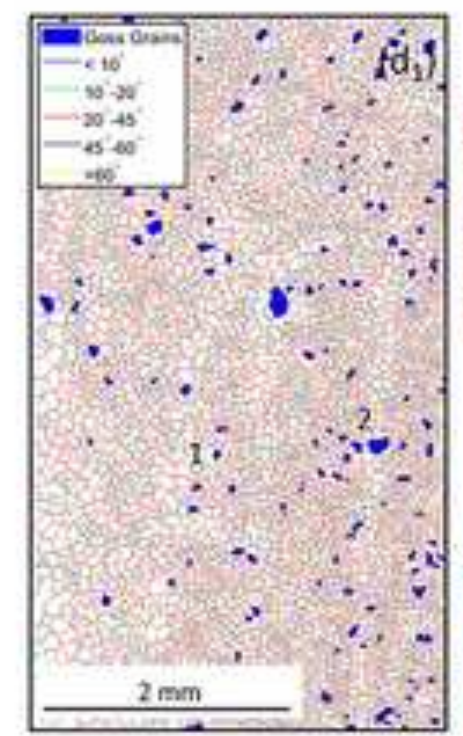

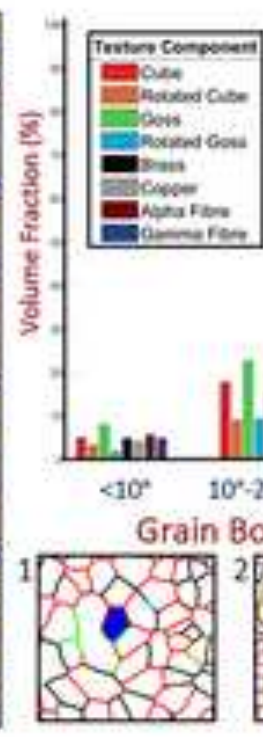

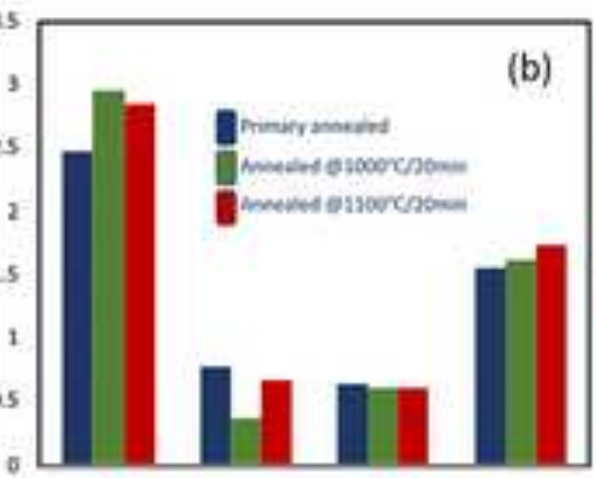

23

57
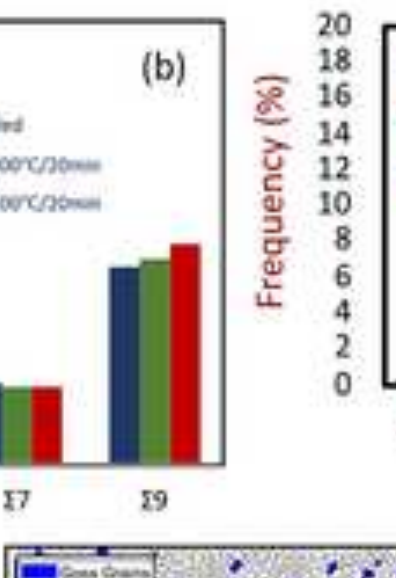

$\left(d_{2}\right)$

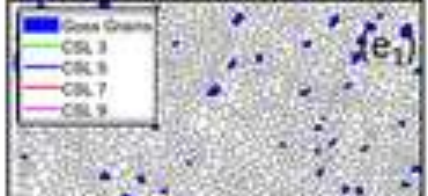

$\therefore$

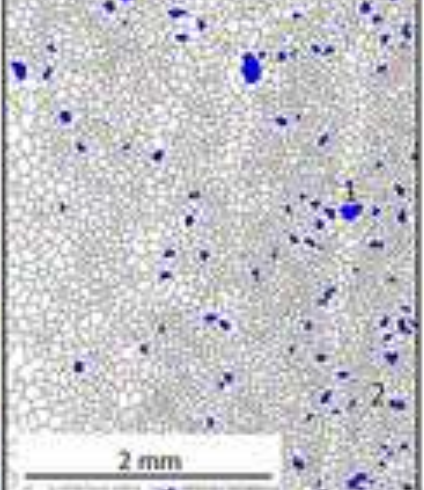

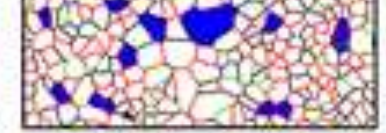

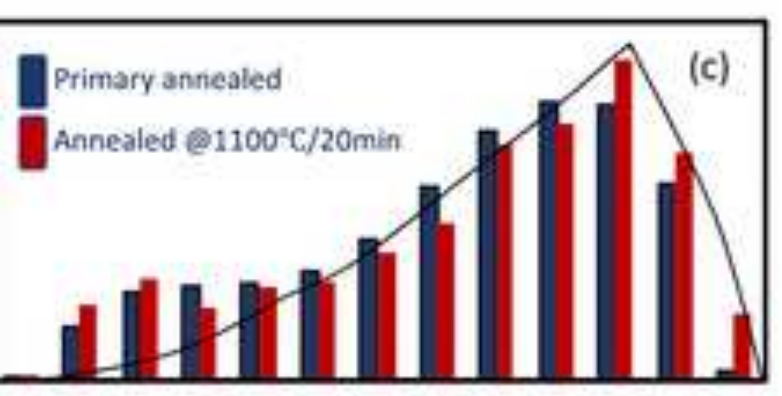

$\begin{array}{lllllllllllll}5 & 10 & 15 & 20 & 25 & 30 & 35 & 40 & 45 & 50 & 55 & 60 & 65\end{array}$ Grain Boundary Angle (")
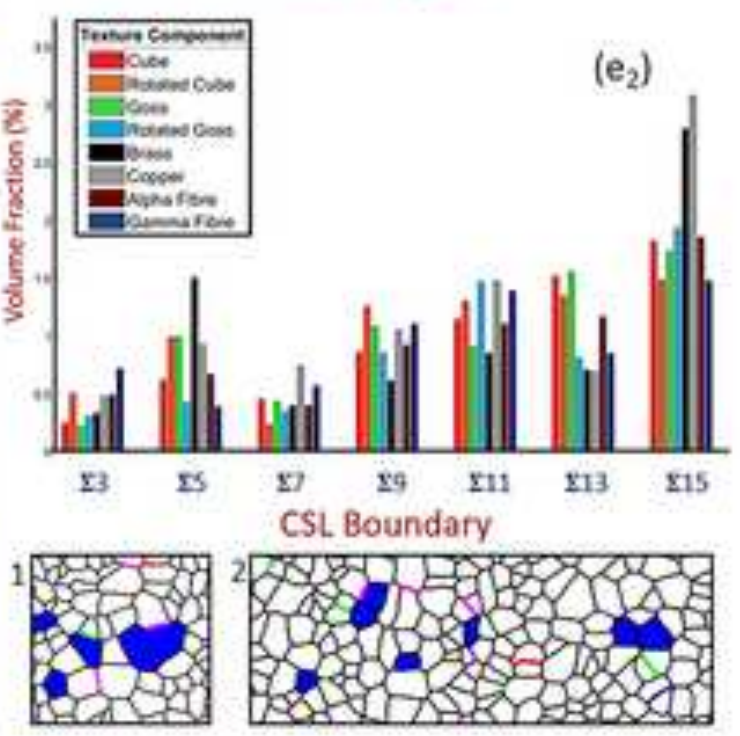

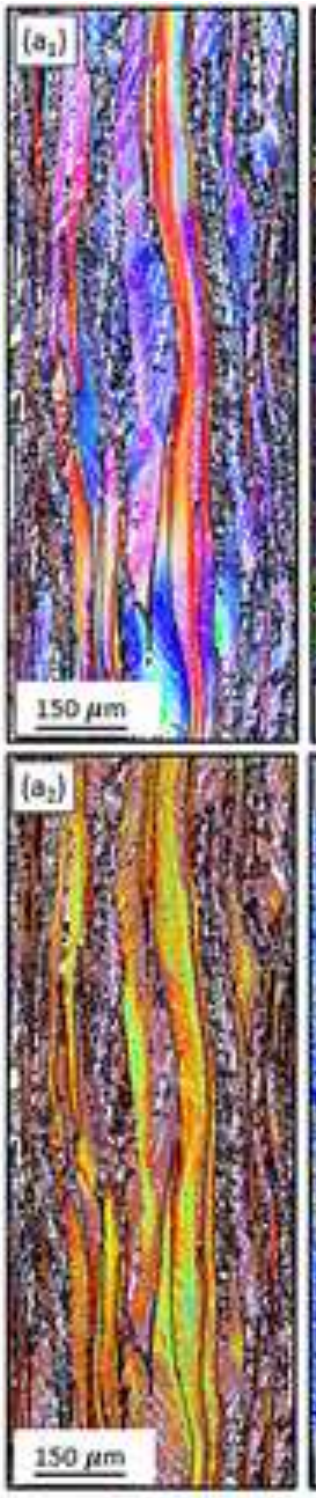
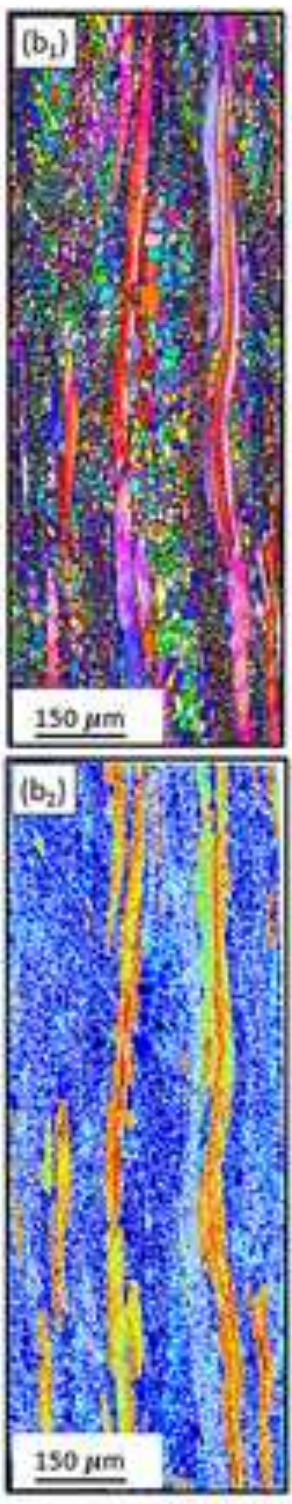
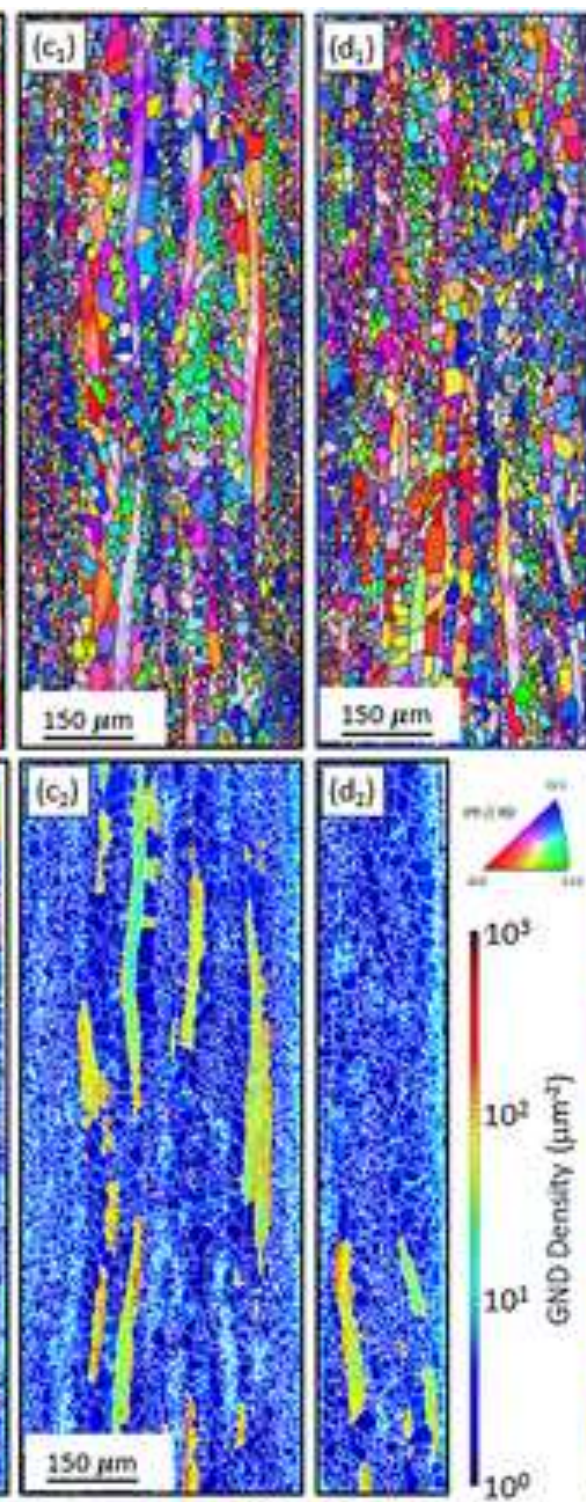

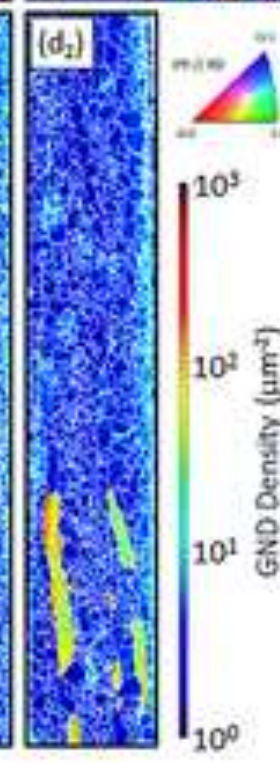

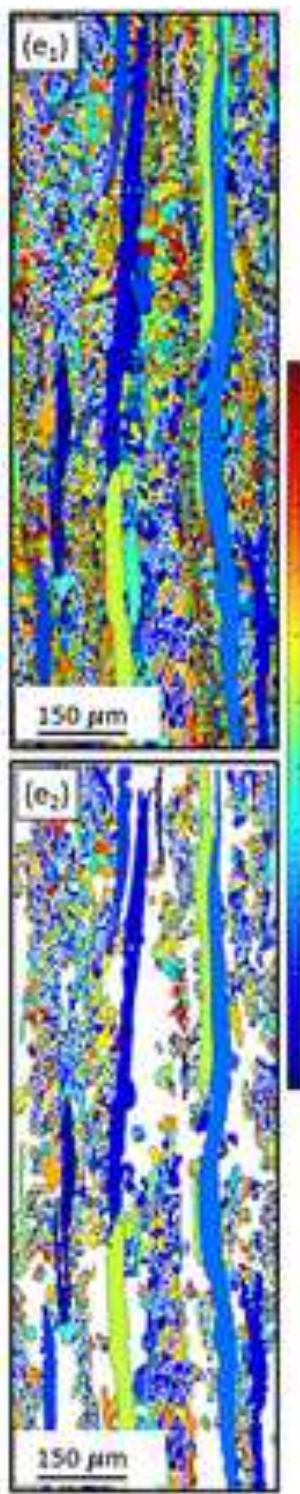

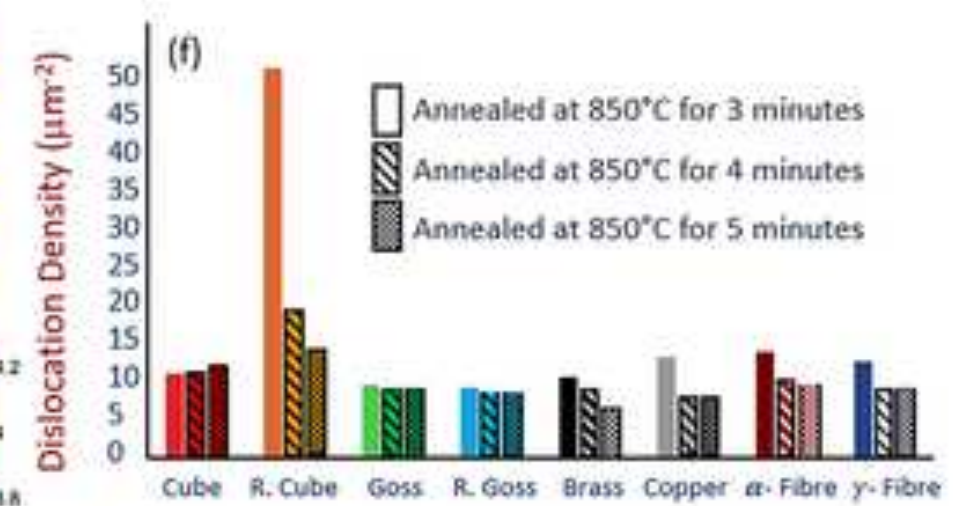

$36 \div 4.5 /(\mathrm{B})$

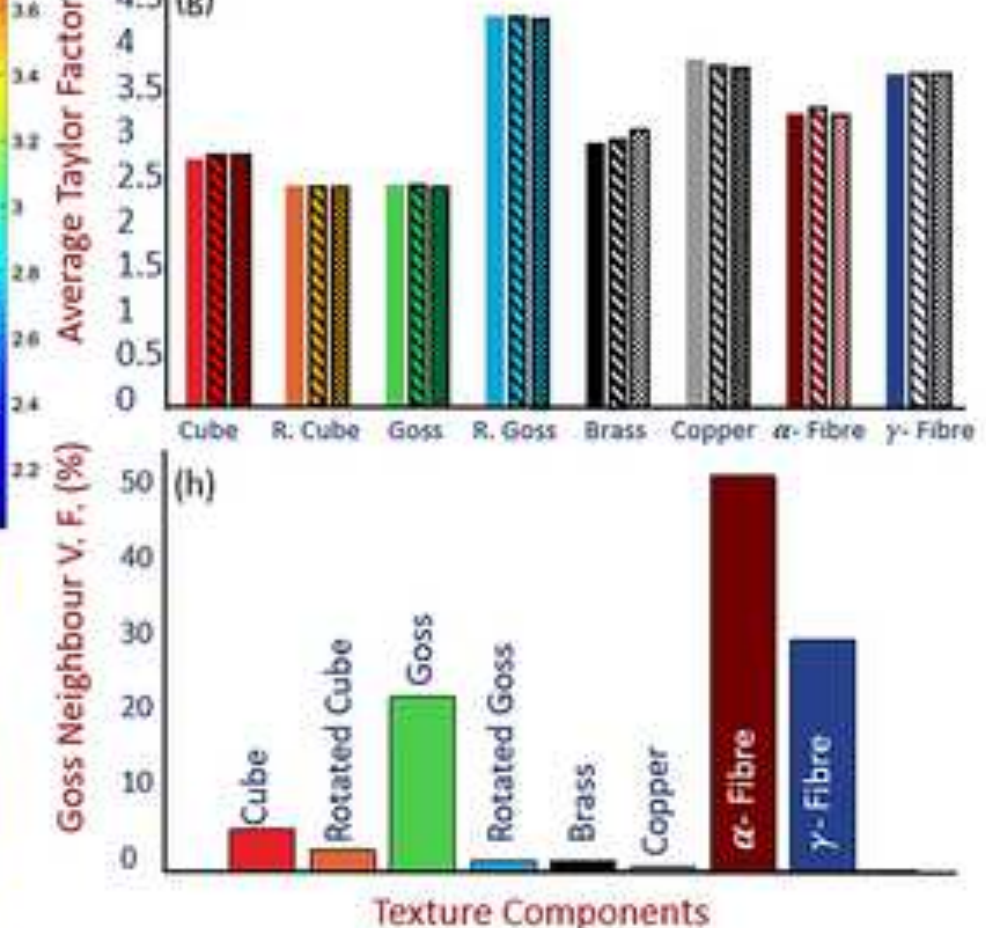

Texture Components 

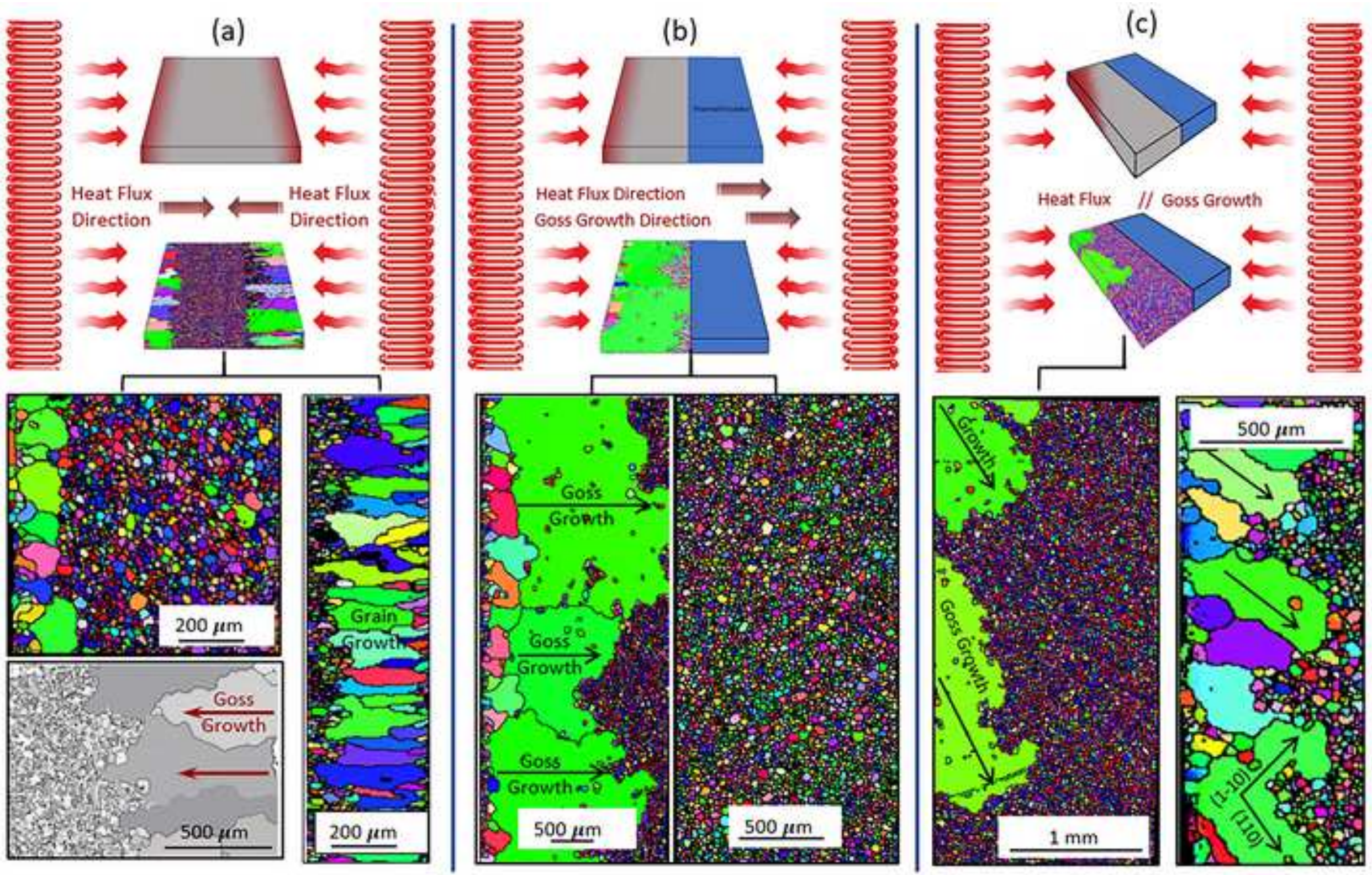

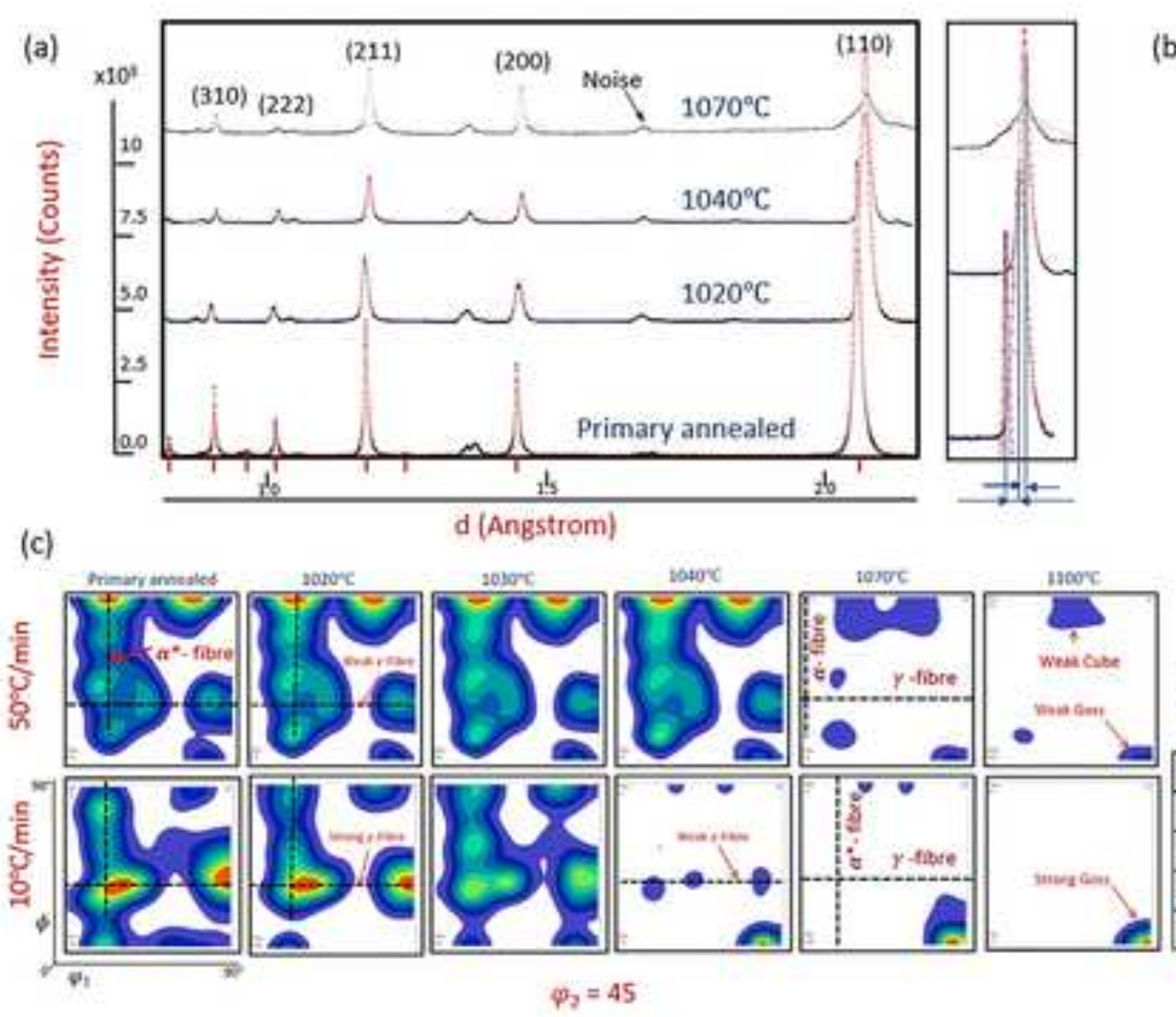

(b)

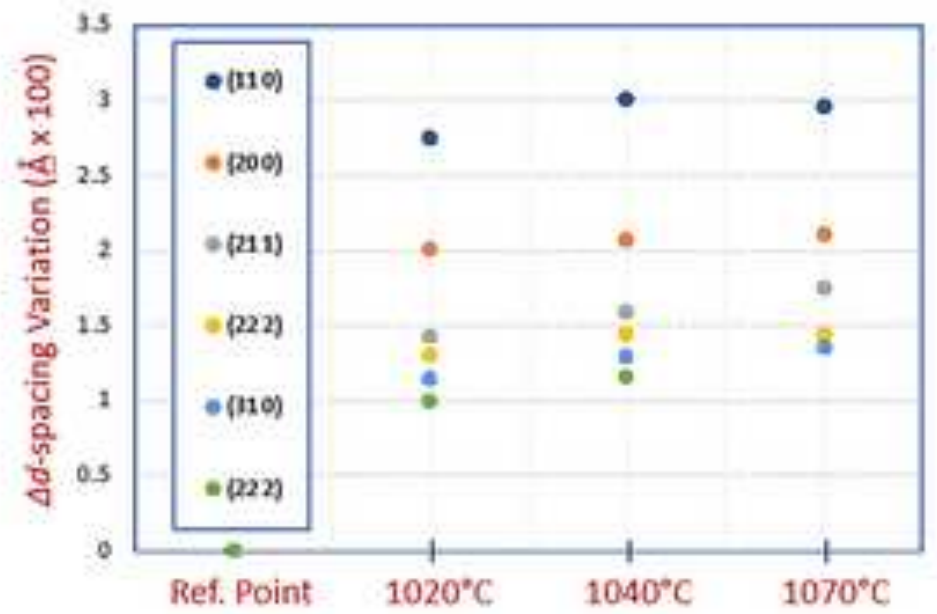

\begin{tabular}{|c|c|c|}
\hline \multirow{3}{*}{$\begin{array}{l}\text { Temp } \\
\text { ("C) }\end{array}$} & \multicolumn{2}{|l|}{ (d) } \\
\hline & \multicolumn{2}{|c|}{ Aln V. E. (ents) } \\
\hline & $50^{\circ} \mathrm{C} / \mathrm{min}$ & $10^{\prime} \mathrm{C} / \mathrm{min}$ \\
\hline RT & 0.0496 & 0.0649 \\
\hline 1020 & 0.0394 & 0,0606 \\
\hline 1040 & 0.0247 & 0.0499 \\
\hline 1070 & 0.00178 & 0.0103 \\
\hline 1100 & 0.00011 & 0.00015 \\
\hline
\end{tabular}

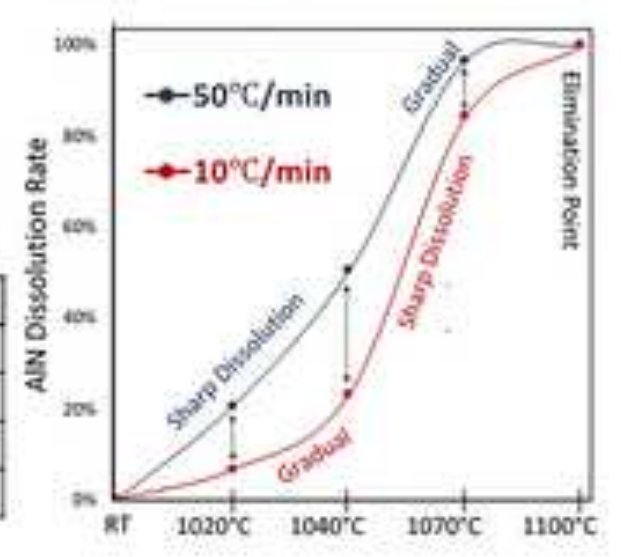


(a)

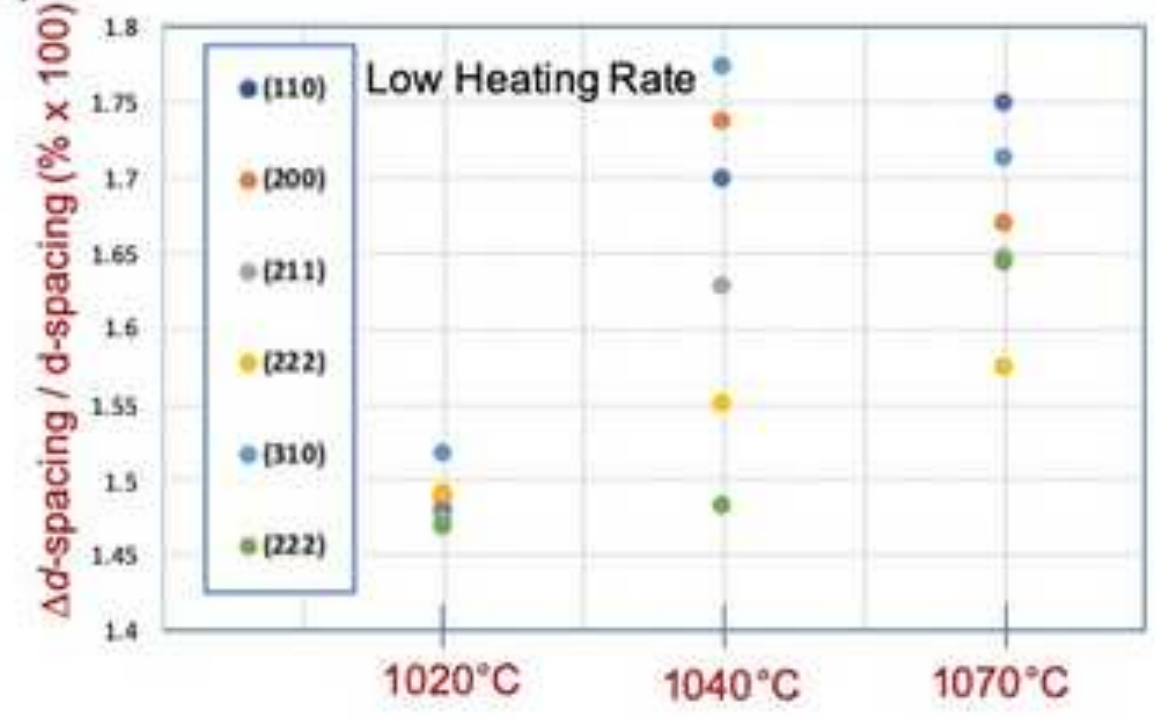

(b)

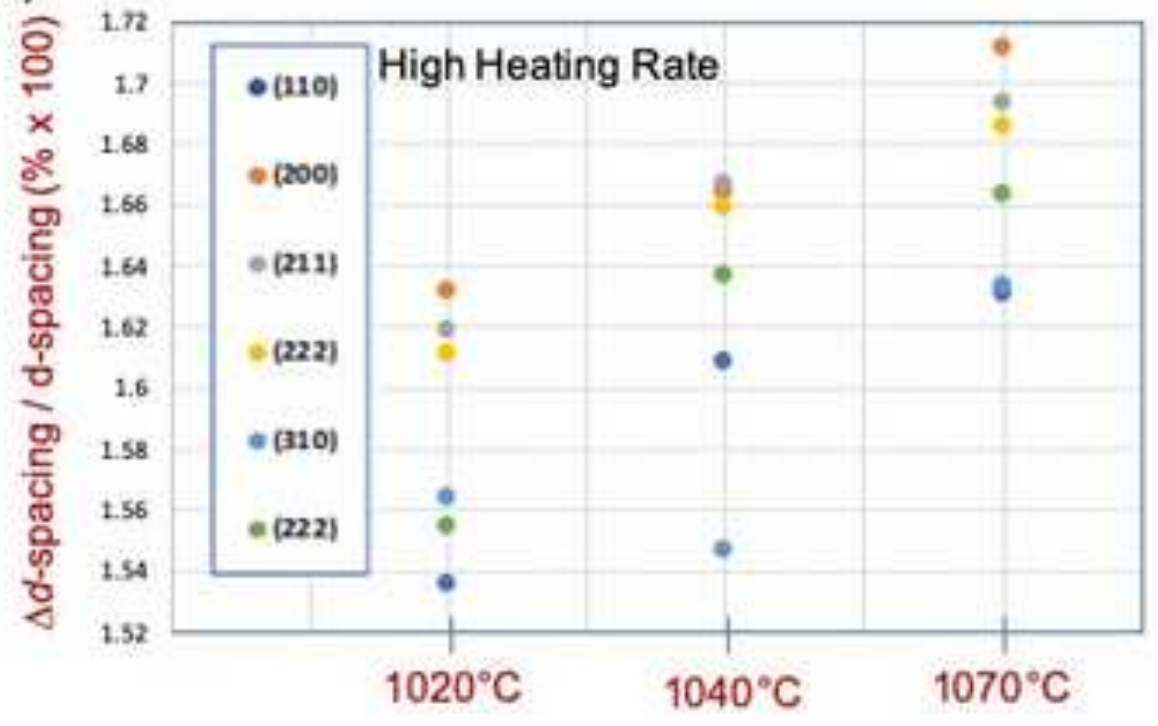




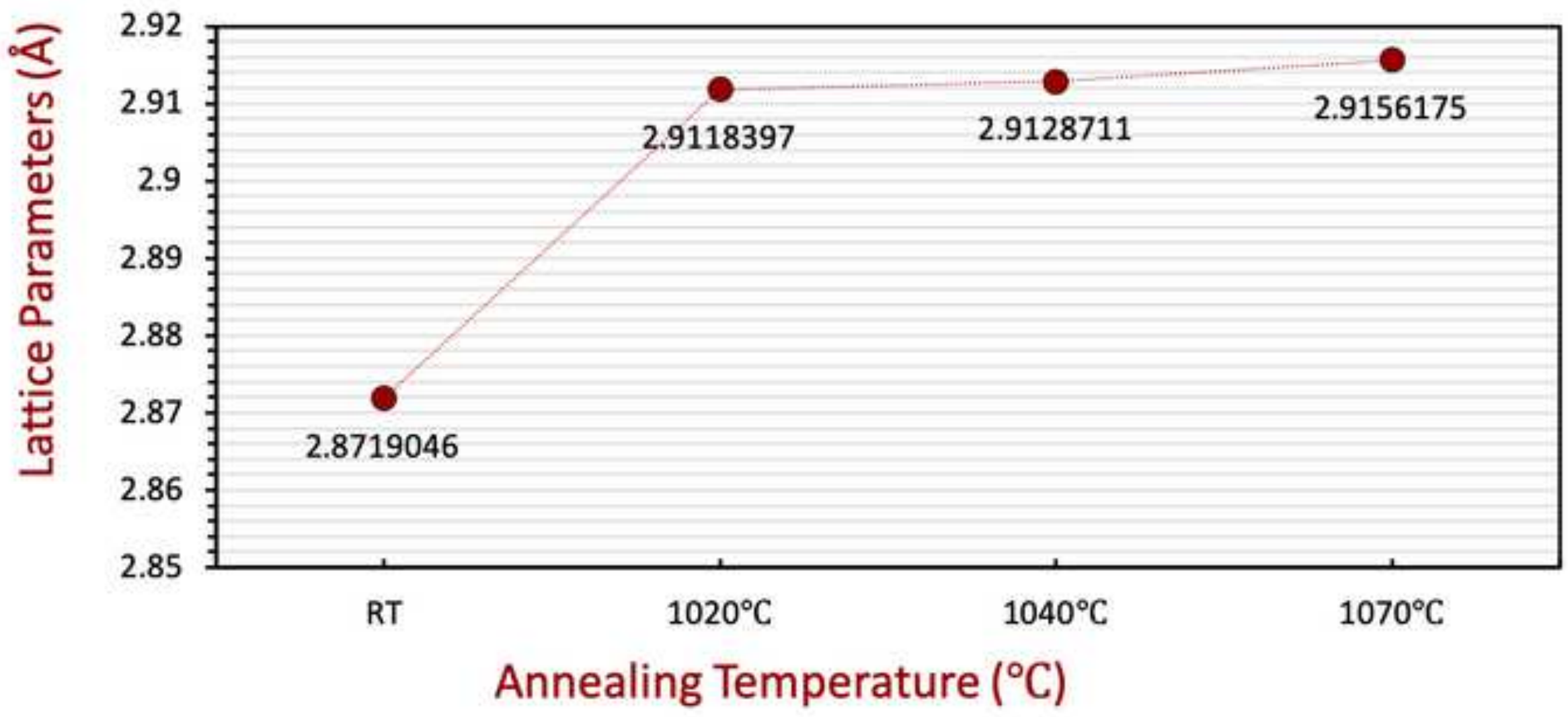



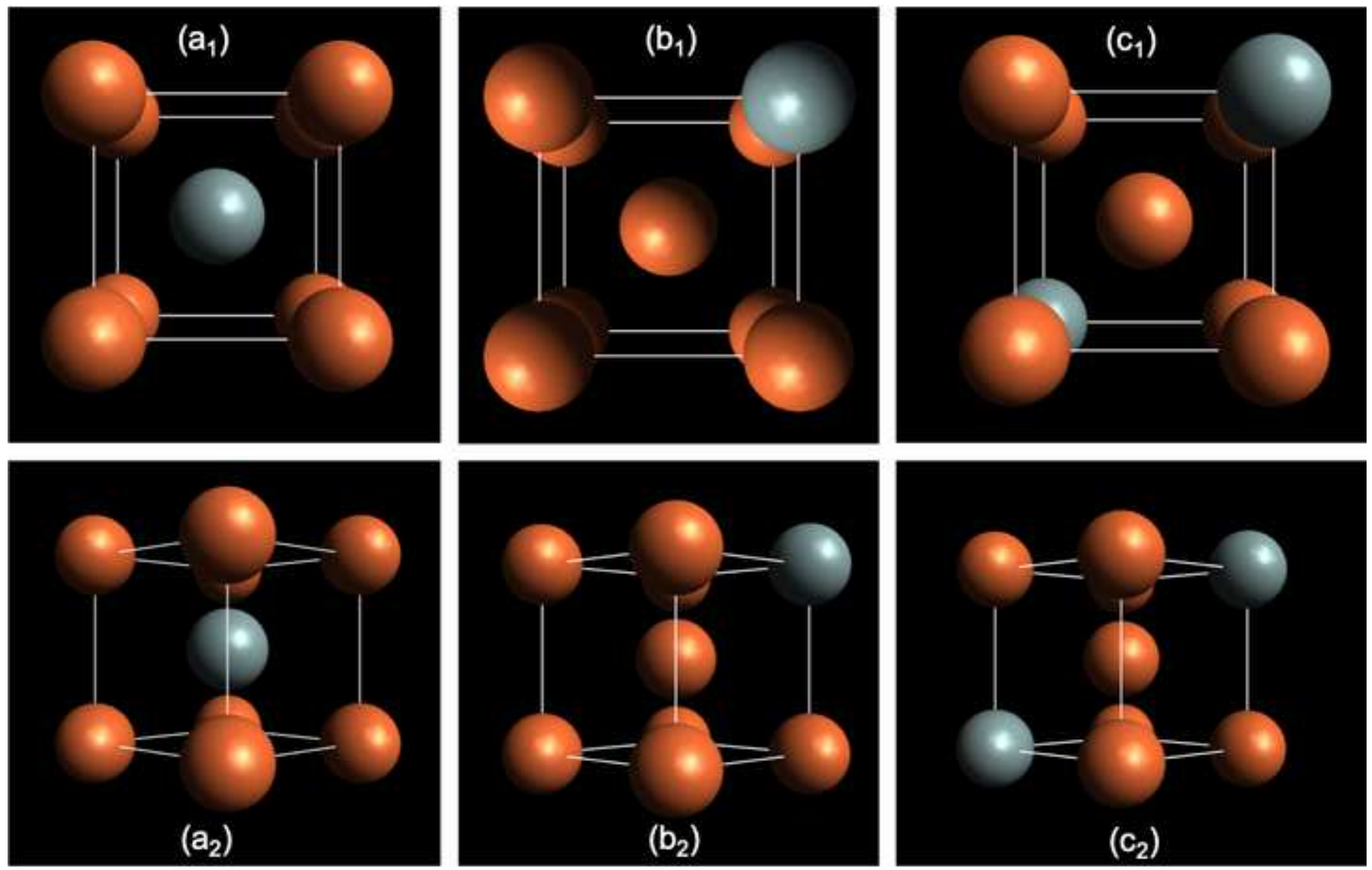

$\left.a_{2}\right)$

$\left(b_{2}\right)$

$\left(c_{2}\right)$ 

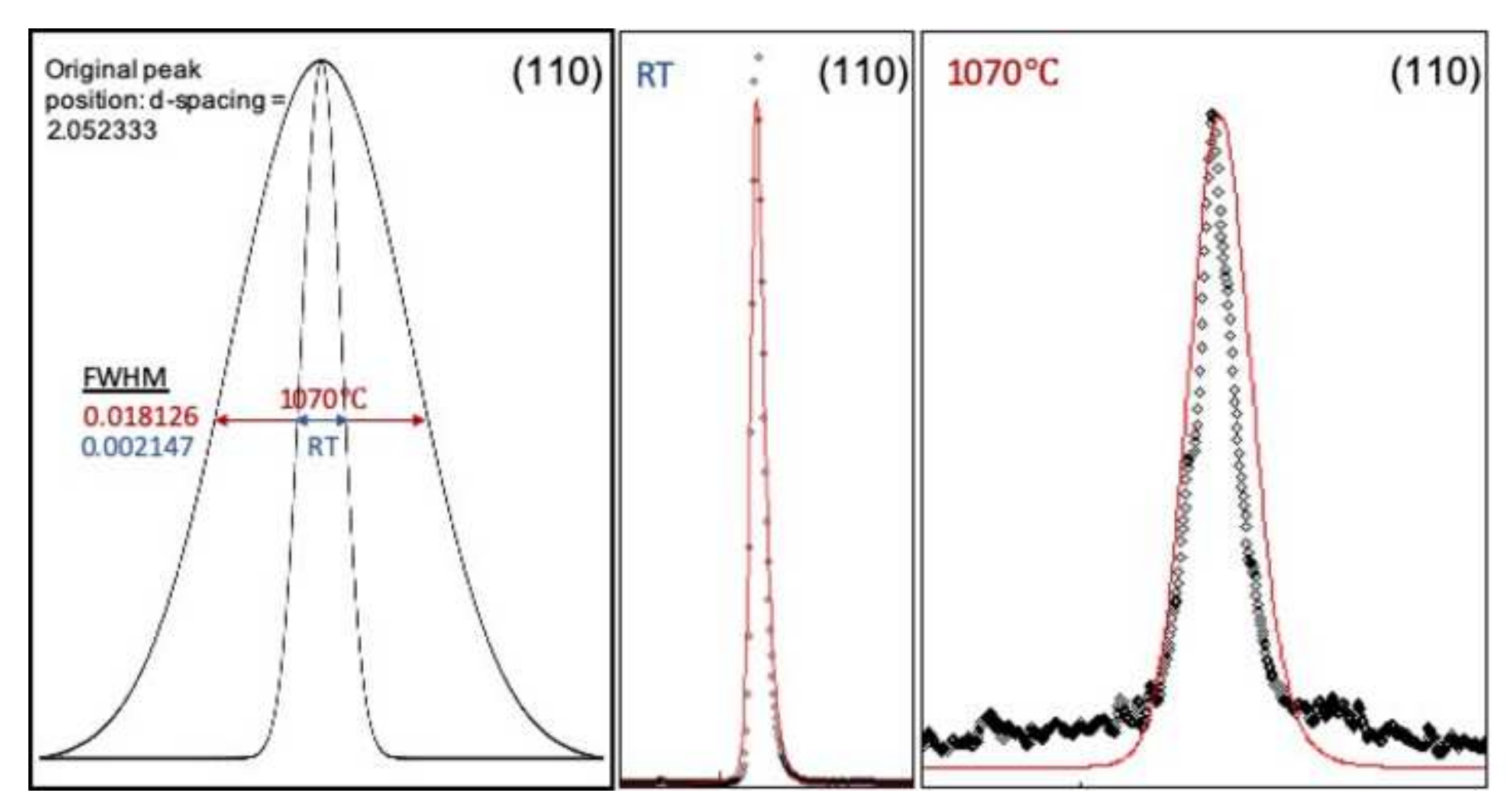


\section{(110)}

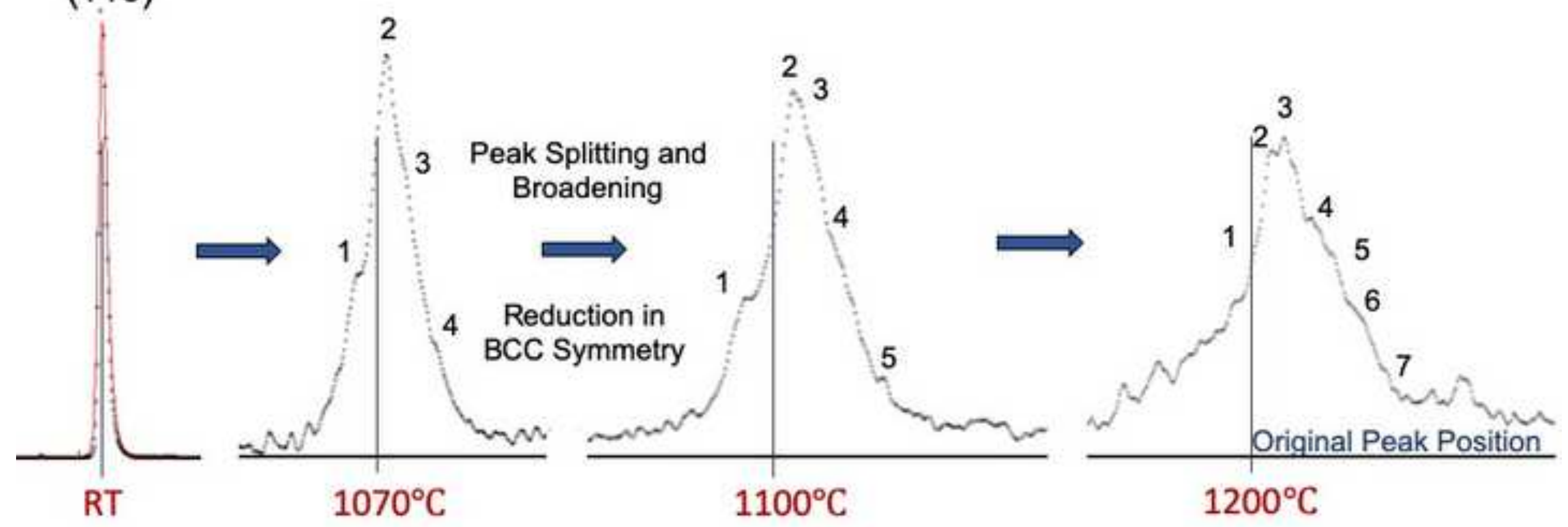




\section{(200)}

3

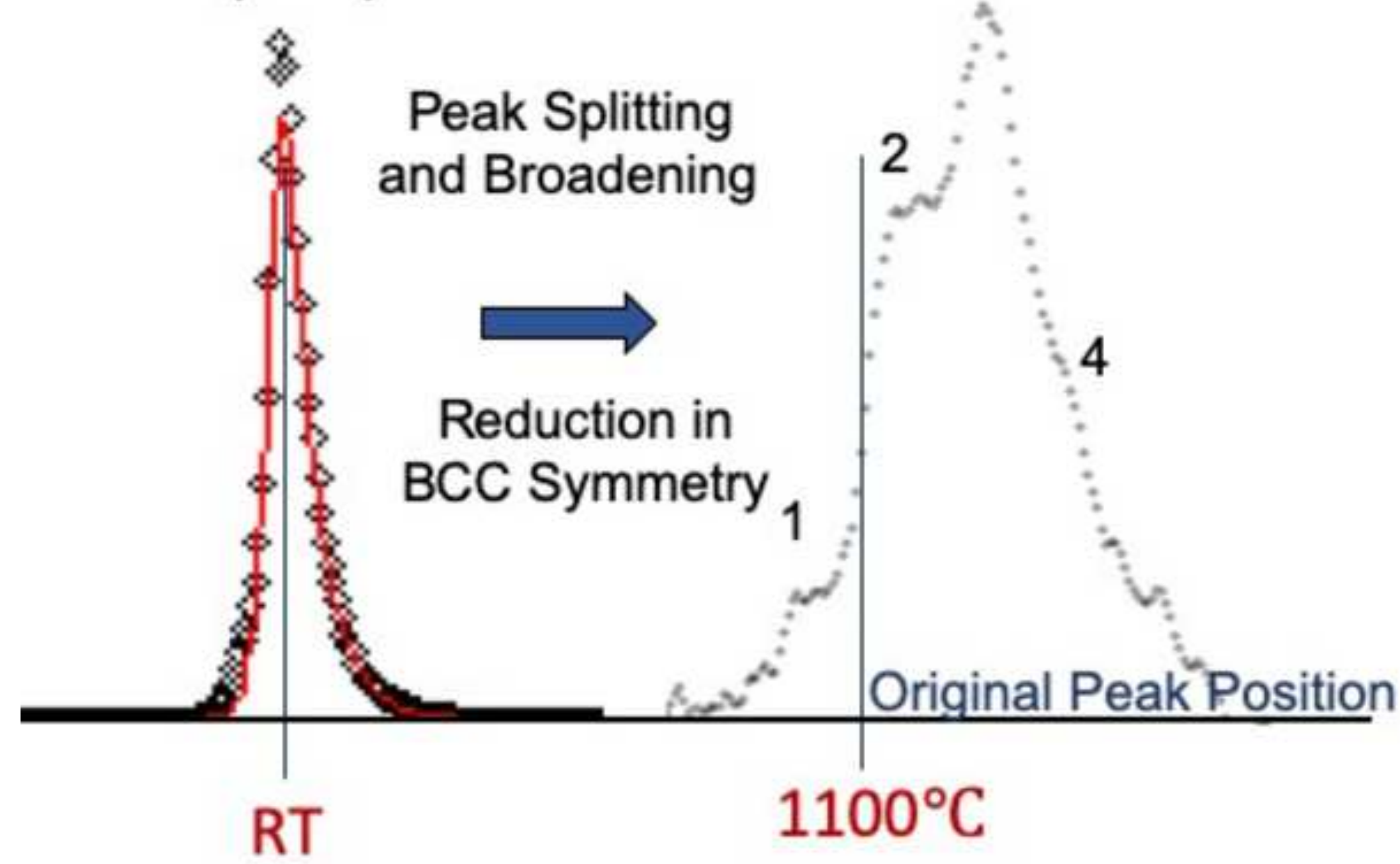




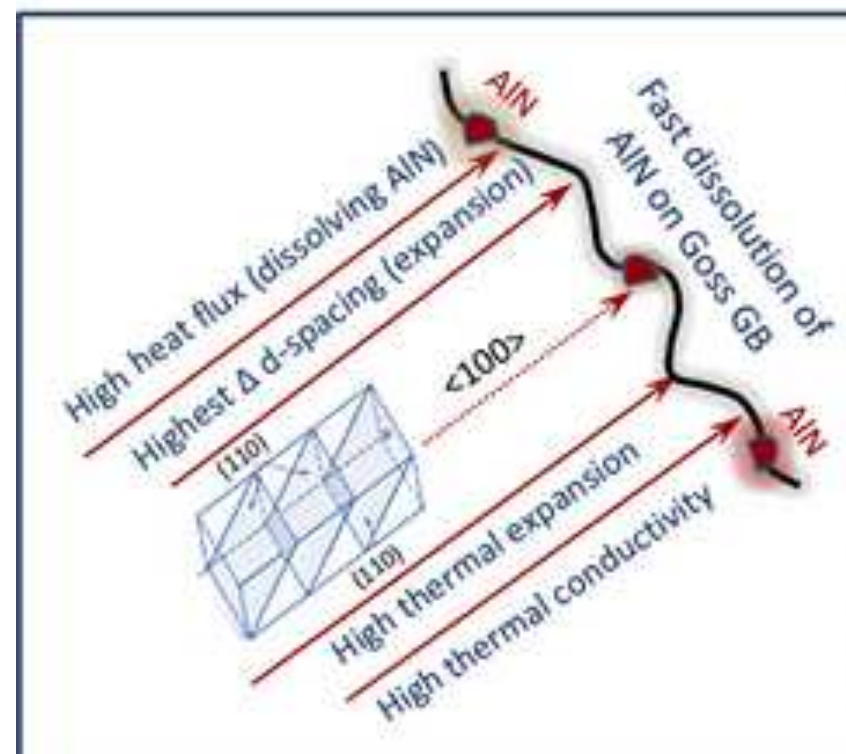

AGG Early Stage

\section{Goss Abnormal Grain Growth Mechanism in 3\% Si Steel}
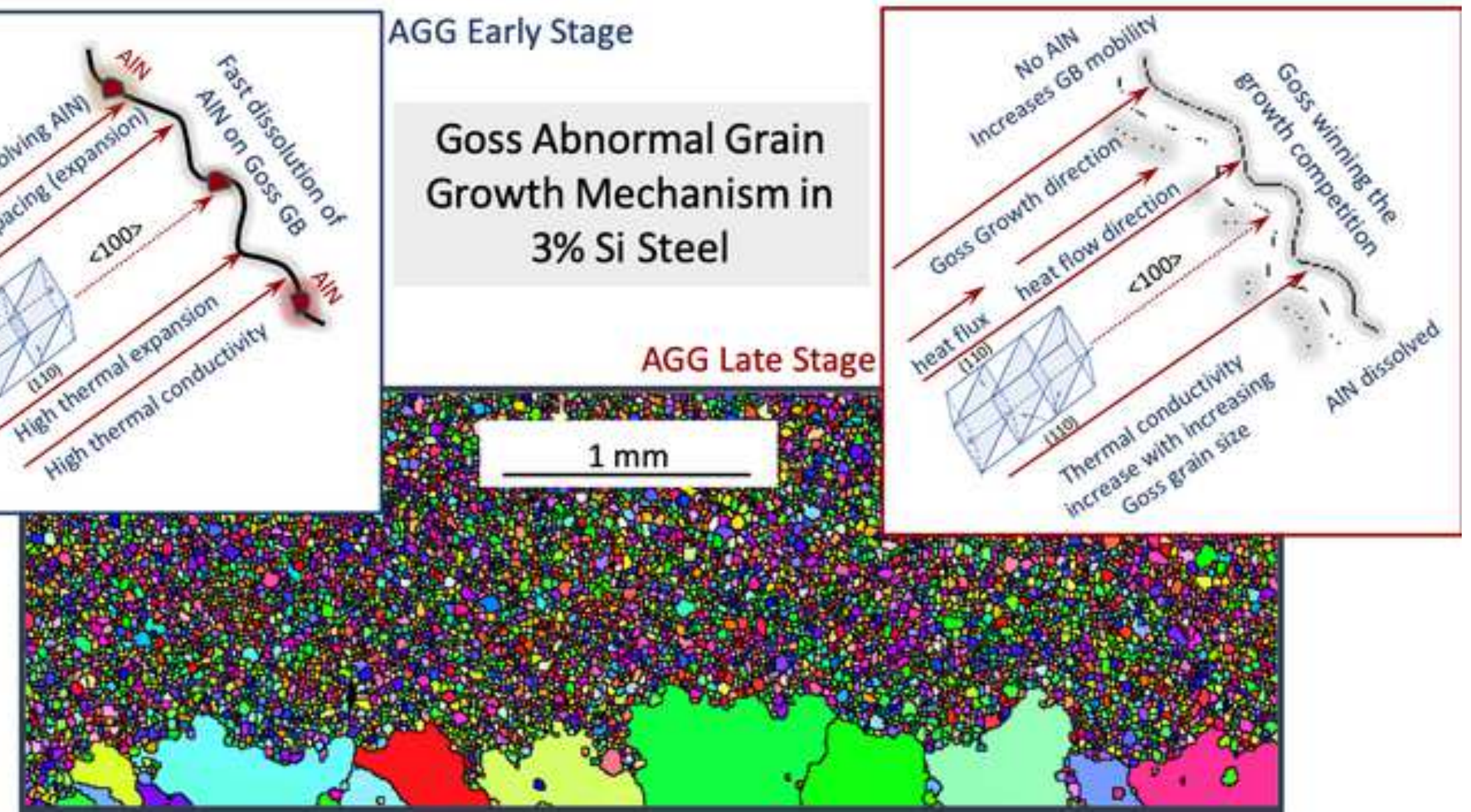


\begin{tabular}{|c|c|c|c|c|c|c|c|c|c|c|}
\hline Si & $\mathbf{C}$ & $\mathbf{N}$ & $\mathbf{M n}$ & $\mathbf{P}$ & $\mathbf{A l}$ & $\mathbf{C u}$ & $\mathbf{N i}$ & $\mathbf{C r}$ & $\mathbf{S n}$ & $\mathbf{P b}$ \\
\hline 3.2 & 0.0017 & 0.0011 & 0.089 & 0.025 & 0.005 & 0.006 & 0.005 & 0.12 & 0.052 & 0.002 \\
\hline
\end{tabular}


Supplementary Material
Click here to download Supplementary Material: S1.jpg

Supplementary Material
Click here to download Supplementary Material: S1.jpg

Click here to download Supplementary Materiat: s1.jpg

(1)

$\sqrt{2}$

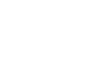

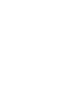

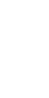

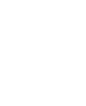

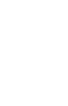

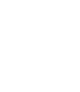

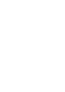

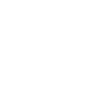

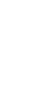

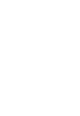
(1)

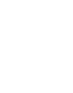

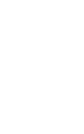

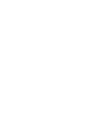

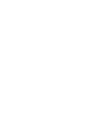
(1)

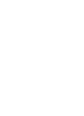

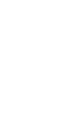

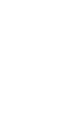

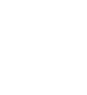
(n)

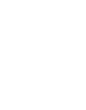
(1) 


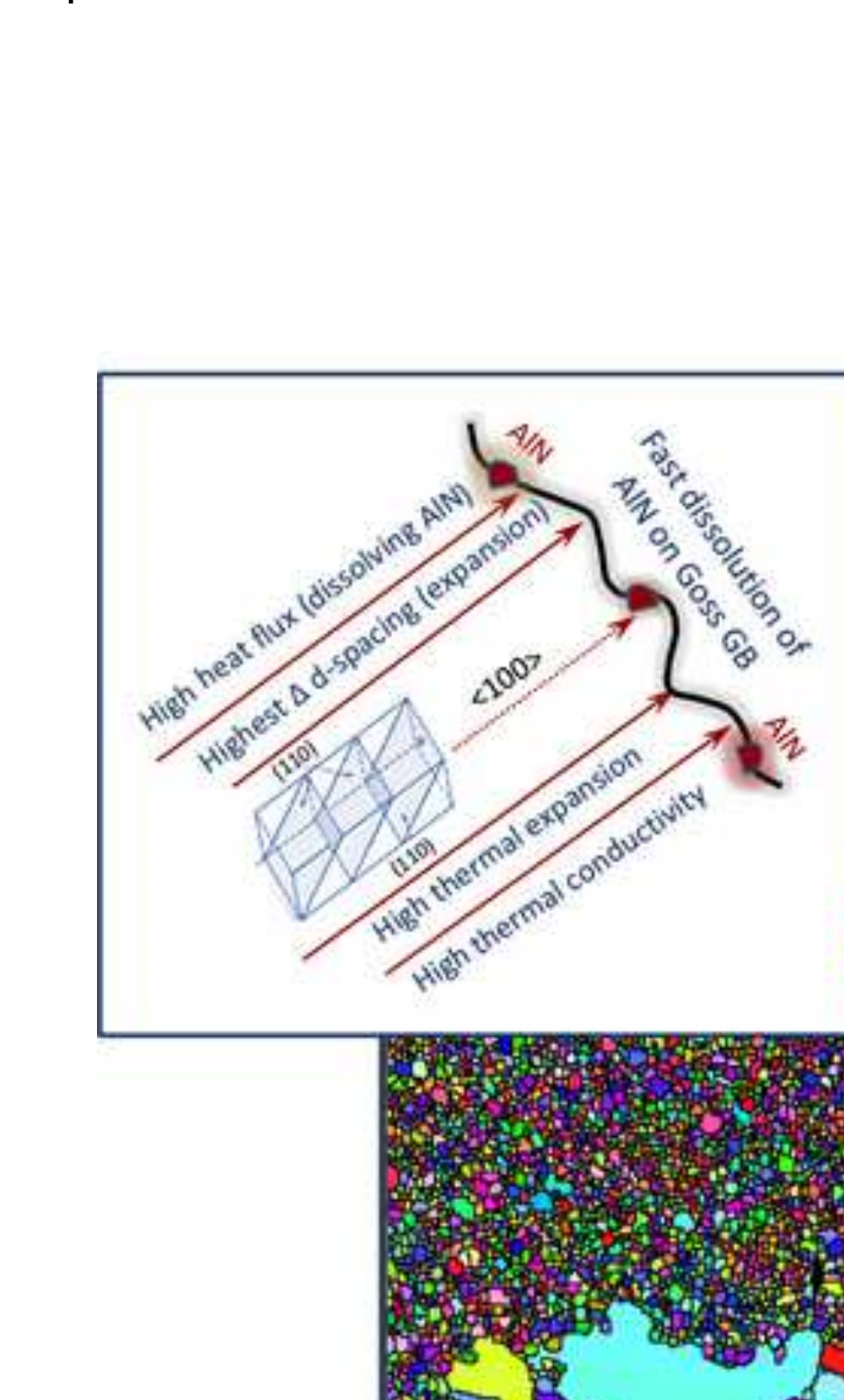

AGG Early Stage

Goss Abnormal Grain Growth Mechanism in $3 \%$ Si Steel
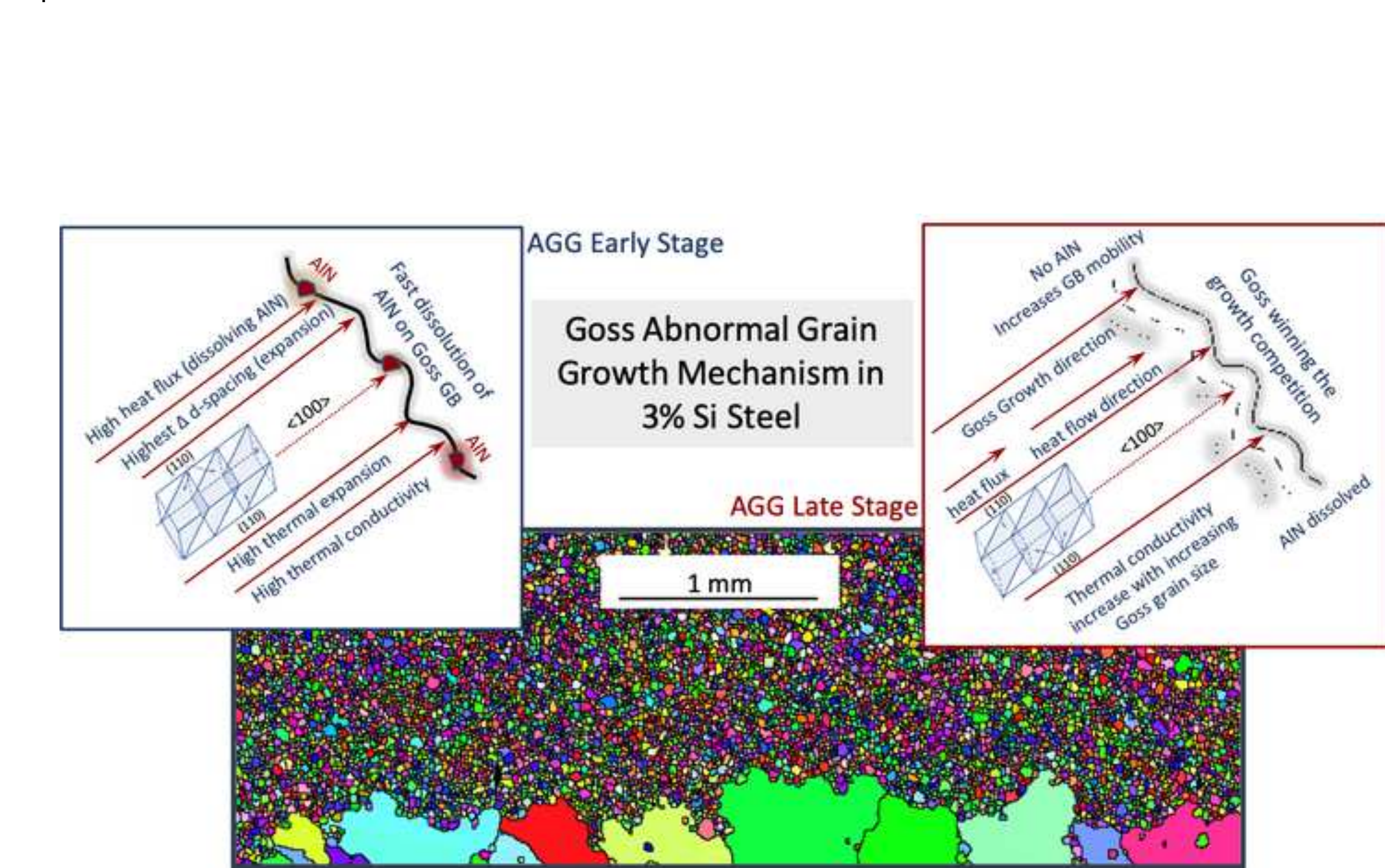

AGG Late Stage

19. When
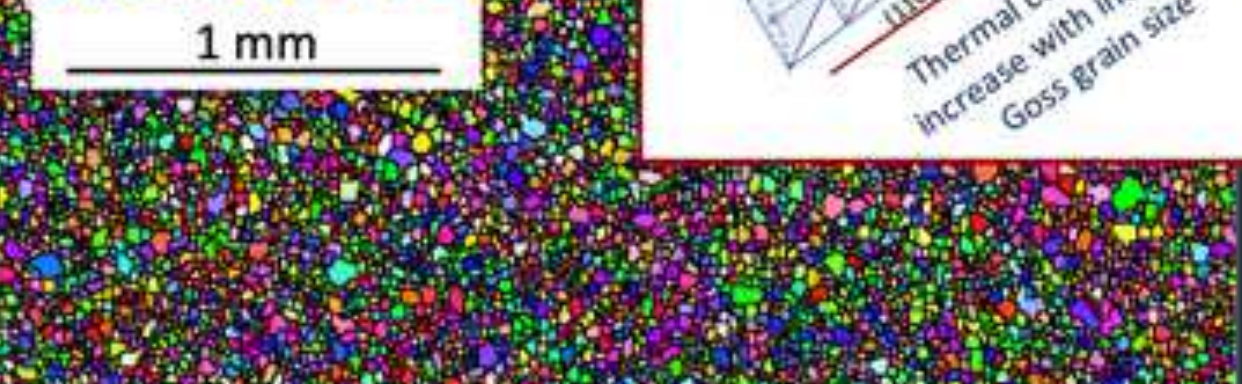

.
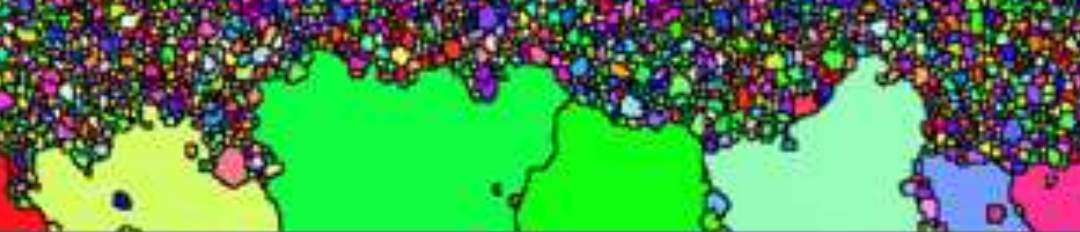


\section{${ }^{*}$ Declaration of Interest Statement}

\section{Declaration of interests}

$\bigotimes$ The authors declare that they have no known competing financial interests or personal relationships that could have appeared to influence the work reported in this paper.

$\square$ The authors declare the following financial interests/personal relationships which may be considered as potential competing interests:

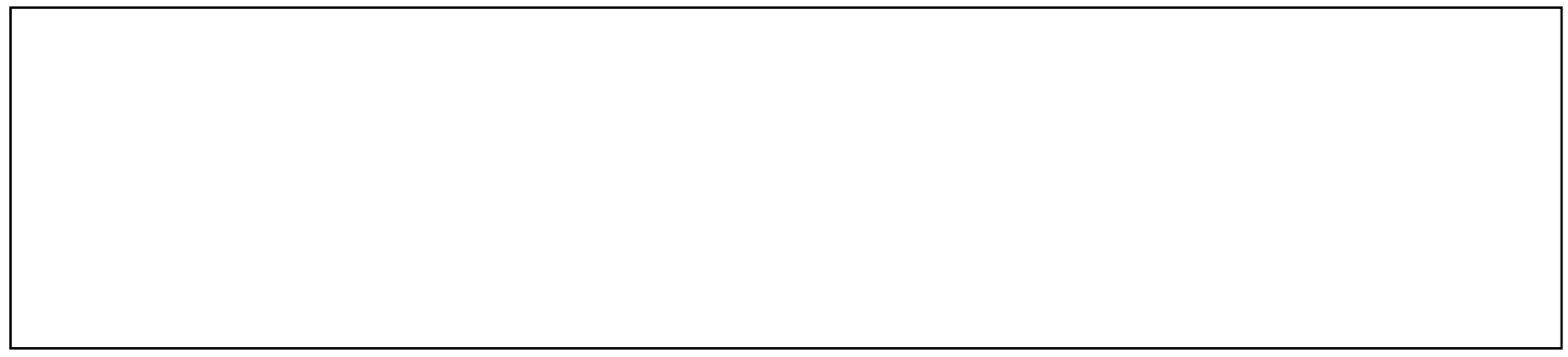

